\title{
The Influence of Rubber Inclusion on the Dynamic Response of Rail Track
}

\section{Yujie Qi, Ph.D., A.M.ASCE; Buddhima Indraratna, Ph.D., F.ASCE²;}

${ }^{1}$ Lecturer, and Program Co-leader of Transport Research Centre, School of Civil and Environmental Engineering, University of Technology Sydney, Sydney NSW 2007, Australia. Email: yujie.qi@uts.edu.au

${ }^{2}$ Distinguished Professor of Civil Engineering, Founding Director of Australian Research Council's Industrial Transformation Training Centre for Advanced Technologies in Rail Track Infrastructure (ITTC-Rail), Director of Transport Research Centre, School of Civil and Environmental Engineering, University of Technology Sydney, Sydney, NSW 2007, Australia. Email: buddhima.indraratna@uts.edu.au

$\dagger$ Author for correspondence:

Yujie Qi

Lecturer \& Program Co-leader of Transport Research Centre, Faculty of Engineering and Information Technology, University of Technology Sydney, Email: Yujie.qi@uts.edu.au 
1 ABSTRACT: Heavier and faster trains have motivated researchers to seek better ways to absorb

2 the increasing amount of energy imparted to rail foundations and mitigate track deterioration. In

3 recent years resilient rubber products have attracted more attention due to the high level of

4 damping and the associated energy absorbing capacity of rubber. However, since rubber granules

5 have lower shear strength and higher compressibility compared to natural rock aggregates, a better

6 understanding of how rubber inclusions can influence the track system is imperative, especially

7 before putting these recycled resilient materials into practice. In this paper, the performance of rail

8 track incorporating an alternative subballast layer, i.e. a synthetic energy absorbing layer (SEAL)

9 consisting of a mixture of granulated rubber and mining waste is evaluated through large-scale

10 prismoidal triaxial tests and a computational dynamic model. It is revealed that the amount of

11 granulated rubber in SEAL composites has a significant influence on the dynamic behaviour of

12 the track. Fundamentally, increasing the amount of rubber within SEAL leads to a higher vertical

13 deformation, increased energy absorbing capacity, and a higher damping ratio and vibration level,

14 while reducing the ballast degradation, track stiffness and lateral movement (dilation) of the track.

15 It has been found that $10 \%$ of rubber by mass is the optimal amount of rubber to be included in

16 SEAL. This amount of rubber will ensure that a ballasted track can efficiently reduce the dynamic

17 contact pressure at the interface between different track layers (i.e. sleeper, ballast, subballast, and

18 subgrade), as well as reduce the lateral spread (dilation) and breakage of ballast without generating

19 excess vibration and settlement comparing to traditional track materials.

20 Keywords: recycled rubber; dynamic loading; ballast degradation; railway foundation; large-

21 scale laboratory tests; track dynamic model. 


\section{Introduction}

Due to the increasing demand for passenger and freight transportation, railways are now operating heavier and faster trains. As a result, the dynamic loads from moving stock lead to higher stresses and exacerbated ground vibration for track substructure, which in return damage the track components, escalate track deterioration (e.g. ballast degradation and track displacement), and increase the risk of derailment. Consequently, more frequent track maintenance is required, which then increases the cost of maintenance. Various techniques have been proposed to mitigate these adverse effects such as improving track foundations by including resilient components and geosynthetics, and attenuating ground vibrations from the source or receiver (Fernandes et al. 2008, Indraratna and Nimbalkar 2013, Tatsuoka et al. 2014, Toward et al. 2014, Fathali et al. 2019). Of these techniques, researchers and practitioners are realising that the inclusion of rubber materials such as recycled tyre cells, under-ballast shock mats, under-sleeper pads, and granulated rubber added to the compacted capping layer or to the ballast later itself can be beneficial options; this is because their higher damping properties and greater energy absorbing capacity help the track system to dissipate the energy from the dynamic (moving) loading and thereby mitigate the damage to rail tracks (Indraratna et al. 2020, Qi and Indraratna 2020).

Previous studies found that installing rubber shock-mats beneath the sleepers or ballast stratum can significantly reduce ballast degradation and the stresses developed at the ballast-sleeper interface, while increasing the damping ratio and energy absorption capacity of the track system (Lakušić et al. 2010, Nimbalkar et al. 2012, Sol-Sánchez et al. 2014, Kaewunruen et al. 2017, Navaratnarajah and Indraratna 2017, Indraratna et al. 2018a, Indraratna et al. 2019, Jayasuriya et al. 2019, Ngo et al. 2019). Xin and Gao (2011) found that installing rubber mats in tracks over a concrete bridge deck reduces the vertical acceleration of the rail by almost $73 \%$ as the train passes 
45 over at high speed (over $250 \mathrm{~km} / \mathrm{h}$ ). Indraratna et al. (2018c) used recycled tyre cells to confine

46 crushed basalt forming the capping layer. Large-scale testing and numerical modelling have shown

47 a reduction in lateral and vertical deformation, as well as lower ballast degradation and higher

48 bearing capacity. Apart from rubber mats/pads and recycled tyre cells, granulated rubber or rubber

49 crumbs have also proven to be alternative options for use in rail tracks. Tyre-derived aggregates

50 mixed with ballast have been proposed by other researchers (Sol-Sánchez et al. 2015, Esmaeili et

51 al. 2017, Gong et al. 2019) to reduce particle breakage and abrasion during tamping and subsequent

52 track operations. Indraratna et al. (2018b) developed a synthetic energy absorbing layer (SEAL)

53 by mixing rubber crumbs with industry by-products (e.g. steel furnace slag and coal wash) used as

54 a subballast layer. The authors have carried out small-scale and large-scale laboratory tests to

55 examine the performance of the SEAL mixture and found that with a proper amount of rubber the

56 SEAL can reduce ballast degradation and maintain acceptable stiffness and deformation (Qi et al.

57 2018b, Qi and Indraratna 2020). Since all these rubber inclusions can be manufactured from waste

58 tyres, the resulting improvements are carbon-friendly and economically attractive.

59 Despite the obvious advantages of using rubber materials, there are still some concerns about 60 including these soft materials in track foundations. In general, the more resilient a track is, the 61 smaller the dynamic wheel load generated from impact will be, which suggests that since the track 62 modulus represents the overall stiffness of the rail foundation, it should not be overly high. 63 However, a relatively low track modulus indicates a softer rail foundation which may lead to 64 ballast or subgrade problems such as extensive deformation and vibration or even "bounciness" 65 (Li and Selig 1995). In fact, when more rubber products are included, the track specimens present 66 a lower track modulus with increasing deformation as reported (i) for a tyre cell reinforced capping 67 layer (Indraratna et al. 2017), (ii) for granulated rubber mixed with ballast (Sol-Sánchez et al. 2015, 
68 Esmaeili et al. 2017), and (iii) for rubber-waste aggregate mixtures used as subballast (Qi et al.

69 2018a). Some soft ballast mats/under sleeper pads can suppress vibration in a relatively high

70 frequency (i.e. $50 \sim 150 \mathrm{~Hz}$ ), but not when the vibration is at a lower frequency (e.g. less than 30

$71 \mathrm{~Hz}$ ) (Kaewunruen and Remennikov 2016). Fernández et al. (2018) found that mixing 2.5-5\% of

72 granulated rubber with ballast reduced peak acceleration in the ballast layer by $20-55 \%$, but the

73 other layers experienced increased vibration. This is why investigating what mechanisms and how

74 much rubber inclusions are needed to optimise the dynamic performance of the track before any

75 further field applications is imperative, especially if the mixtures are to incorporate granulated

76 rubber or rubber crumbs.

77 This paper focuses on the application of granulated rubber/rubber crumbs in rail tracks. It is a 78 continuation of previous studies by the authors on the synthetic energy absorbing layer (SEAL) as 79 a replacement for traditional subballast materials. Previous studies investigated the small scale 80 static and cyclic loading behaviour of the SEAL matrix with different amounts of rubber, and they 81 recommended that $10 \%$ of rubber can ensure an acceptable shear strength while enhancing the 82 damping or energy absorbing properties (Indraratna et al. 2018b, Qi et al. 2019b, Indraratna et al. 83 2020). Qi and Indraratna (2020) also proposed an energy-based analysis which indicated that 10$8413 \%$ rubber will dissipate the accumulated energy through an acceptable deformation in the SEAL 85 layer and reduce ballast breakage. However, how the amount of rubber in a SEAL matrix can 86 influence the dynamic performance of track (e.g. track modulus, damping property, vibration and 87 dynamic loads at the interfaces between each layer) has not yet been addressed. Therefore, in this 88 paper, the influence of the amount of rubber in SEAL on the dynamic response of track is 89 investigated based on a series of large-scale laboratory testing in comparison with traditional track 
90 specimens. The displacement and acceleration of rail influenced by the amount of rubber within a

91 SEAL matrix are also examined utilizing using a track dynamic model.

\section{Large scale physical model for SEAL}

93

94

95

96

97

98

99

100

101

102

103

104

105

106

107

108

109

110

111

\subsection{Materials and test program}

A large-scale physical model of the track was used to carry out a series of prismoidal triaxial tests to investigate the behaviour of the track that incorporates the SEAL matrix. This physical model has a ballast layer on top, a subballast layer in the middle and structural fill at the bottom, which simulates field conditions. The subballast layer was compacted with traditional subballast materials or the SEAL matrix containing varying amounts of rubber. These materials came from local suppliers, i.e., Bombo Quarry (NSW, Australia) for ballast, conventional subballast and structural rockfill, Australian Steel Milling Services for steel furnace slag (SFS), Illawarra Coal for coal wash (CW), and Tyre Crumbs Australia for rubber crumbs (RC). The grading curves of these materials are shown in Fig. 1.

The SEAL matrix was prepared by firstly mixing steel furnace slag with coal wash at a blending ratio of 7:3 by mass to ensure the mixture had sufficient strength while preventing the unacceptable swell pressure indicated by Indraratna et al. (2018b) and Qi et al. (2019b). Different rubber contents $\left(R_{b}=0,10,20,30\right.$ and $\left.40 \%\right)$ were added to the mixture and blended thoroughly to form the following SEAL matrices, i.e., SEAL0, SEAL10, SEAL20, SEAL30 and SEAL40, where the number immediately following the word 'SEAL' denotes the rubber content by weight. The rubber content is limited to $40 \%$ because a mixture with $R_{b}>40 \%$ will have a skeleton dominated by rubber, which is not applicable for civil engineering (Youwai and Bergado 2003). The grading curves for SEAL mixtures with different amounts of rubber are shown in Fig. 1. 
112 The large-scale prismoidal triaxial facility (Fig. 2a) was used to examine the performance of the 113 physical track model that incorporates SEAL. The testing chamber has a plan area of $114600 \mathrm{~mm} \times 800 \mathrm{~mm}$ and a depth of $600 \mathrm{~mm}$. Structural fill to a depth of $100 \mathrm{~mm}$ was compacted 115 to the field dry density $\gamma_{d}$ of $21.4 \mathrm{kN} / \mathrm{m}^{3}$ at the bottom of the test chamber. The subballast layer 116 (either traditional material or a SEAL matrix with different amounts of rubber) on top of the 117 structural fill was compacted to a depth of $150 \mathrm{~mm}$ with the dry density achieving $95 \%$ of the 118 maximum dry density. The maximum dry density $\gamma_{d, \max }$ of traditional subballast material is $11918.5 \mathrm{kN} / \mathrm{m}^{3}$ with the optimum moisture content (OMC) of $4.5 \% ; \gamma_{d, \max }$ of SEAL mixtures varies 120 from 20.3 to $12.4 \mathrm{kN} / \mathrm{m}^{3}$ as the rubber content increases from 0 to $40 \%$, and OMC of SEAL 121 mixtures remains within the range of 8-11\%. A $200 \mathrm{~mm}$ thick layer of ballast with a bulk density 122 of $15.3 \mathrm{kN} / \mathrm{m}^{3}$ was then prepared on top of the subballast layer. A rail-concrete sleeper assembly 123 with stiff E-type clip fastener system was placed on top of the ballast and then it was filled and 124 levelled with shoulder ballast. A pressure cell was installed on top of each layer to detect the 125 dynamic load (Fig. 2b). The ballast directly beneath the sleeper was painted for visual examination 126 of breakage and collected after each test to determine the ballast breakage index (BBI).

127 The cyclic loading was applied with a loading frequency of $15 \mathrm{~Hz}$ and the maximum vertical stress 128 under the sleeper was $q_{\max }=230 \mathrm{kPa}$ (Fig. 2c). This was to simulate the typical field conditions 129 for an Australian freight train having a 25-tonne axle load running at a maximum speed of 110 $130 \mathrm{~km} / \mathrm{h}$ (Indraratna et al. 2018c, Navaratnarajah et al. 2018). Before each test, a conditioning phase 131 with a loading frequency of $5 \mathrm{~Hz}$ over 100 cycles was applied to increase the contact area between 132 the sleeper and the underlying ballast. During testing, the two sidewalls in the test chamber that 133 are parallel to the sleeper were kept still, while the other two sidewalls (i.e. perpendicular to the 134 sleeper) were allowed to move laterally under the confining pressure of $15 \mathrm{kPa}$. This was in order 
135 to simulate the condition of plane strain where deformation in the longitudinal direction of the

136 track could be ignored. Each test was continued until $N=500,000$ cycles, and there were six tests

137 in total during which the subballast layer was composed of either traditional subballast or with the

138 SEAL matrix $\left(R_{b}=0,10,20,30\right.$ or $\left.40 \%\right)$.

\subsection{Deformation behaviour}

140 The vertical displacement of the track specimen with the SEAL matrix and traditional subballast

141 materials under cyclic loading is shown in Fig. 3a. As the amount of rubber $\left(R_{b}\right)$ increases in the

142 SEAL matrix the vertical displacement of the test specimen increases because of the increasing

143 compressibility of the SEAL matrix as more rubber is added (Qi et al. 2018b). The vertical

144 deformation of the test specimen increases rapidly in the first few thousands of cycles and then

145 gradually stabilises as the accumulation rate of the vertical strain $\left(\varepsilon_{1}\right)$ decreases with the increasing

146 number of loading cycles (Fig. 3b). Here, the accumulation rate of $\varepsilon_{1}$ denotes the tangential slope

147 of the total vertical strain versus loading cycles plot for two adjacent concerned points. Fig. 3b

148 shows that there is a sharp reduction in the accumulation rate of vertical strain after it reaches $10^{-8}$,

149 which indicates that the increase in vertical deformation is negligible; in this case, the test specimen

150 has apparently attained a state of 'plastic shakedown', which refers to a phenomenon where the

151 granular aggregates under cyclic loading achieve a compacted assembly showing negligible

152 vertical strain increment upon further loading (Lackenby et al. 2007). Except for SEAL40, the

153 vertical deformation of all the test specimens attains plastic shakedown, albeit the specimen with

154 traditional materials and SEAL0 reach plastic shakedown at around $\mathrm{N}=100,000$ whereas others

155 attain this condition at a later stage $(\mathrm{N}=300,000-400,000)$. Also note that the specimen with

156 SEAL40 begins with a high accumulation rate of vertical strain $\left(>10^{-4}\right.$, Fig. $\left.4 \mathrm{~b}-3\right)$ and fails at 
157 around $\mathrm{N}=1500$ showing substantial settlement (> $40 \mathrm{~mm}$ ) and pronounced vibration, which could 158 be taken as plastic collapse.

159 Lateral displacement and the accumulation rate of lateral strain of all the test specimens are shown 160 in Fig. 4. As expected, the lateral dilation of the track specimens decreases as more rubber is added 161 to the SEAL matrix, but only for values of $R_{b}<20 \%$ (Fig. 4a). Except for the specimen with 162 SEAL40, around $70 \%$ of the lateral dilation of other test specimens accumulates in the first 10,000 163 cycles (Fig. 4a) where the accumulation rate of the lateral strain begins at a magnitude of $10^{-6}$ 164 and then gradually drops to a negligible level of $10^{-8}$ (Figs. 4b-1\&2). It is noteworthy that when $165 R_{b} \geq 20 \%$ the lateral displacement of the test specimen fluctuates with increasing loading cycles 166 (Fig. 4a) as the accumulation rate alternates between negative and positive values (Fig. 4b-2). This 167 occurs because when $R_{b} \geq 20 \%$ the skeleton of the SEAL mixture is increasingly dominated by 168 rubber, so the specimen tends to behave as rubber-like (Qi et al. 2018a, Qi et al. 2018c). For the 169 test specimen with SEAL40, the accumulation rate of lateral stain is much higher at $10^{-3}$ than 170 those specimens with smaller amounts of rubber (Fig. 4b-3); this means that lateral dilation has 171 accumulated at a faster rate. Moreover, the changing sign of the accumulation rate for the specimen 172 with $R_{b} \geq 20 \%$ indicates an unstable lateral behaviour because lateral compression and dilation 173 appear alternately (Fig. 4b-3).

174 The elastic vertical deformation of the test specimen shown in Fig. 5a is a good indicator of vertical 175 vibration under cyclic loading (Qi and Indraratna 2020). It is noted that the elastic vertical 176 deformation increases with the loading cycle and stabilises rapidly after 500 cycles. As the $R_{b}$ in 177 the SEAL matrix increases, the elastic vertical deformation of the track specimen increases. The 178 elastic vetical deformation reflects the way of rubber-soil mixtures to release the energy via 179 increased bounciness (up and down movement), hence inducing more vibration in the test 
specimen. In fact, the vertical displacement almost doubles when $R_{b}$ increases from $30 \%$ to $40 \%$.

Fig. $5 \mathrm{~b}$ shows how the final elastic and plastic vertical deformations of the track specimen vary depending on the amount of rubber. The figure shows that elastic and plastic vertical deformations increase as more rubber is added to SEAL, and when $R_{b}$ increases from $30 \%$ to $40 \%$ this associated rapid increase in vertical deformation (settlement) corroborates with the severely increased vibration observed in the specimen with SEAL40. It is easy to understand that more rubber will cause more elastic strain as rubber is a visco-elastic material. On the other hand, as more rubber is added in SEAL, the mixture tends to present an increasingly looser condition under the same compaction effort, meaning that the mixture has a larger void space within the granular assembly (Indraratna et al. 2018b, Tawk et al. 2020). This will then enable further compaction under continuously dynamic loading, hence causing a higher plastic deformation.

Compared to the test specimen tested here with conventional subballast materials and the specimen tested under the same loading conditions by Navaratnarajah et al. (2018), the specimen with a SEAL matrix having $R_{b} \leq 10 \%$ has an acceptable settlement $(7.2-11 \mathrm{~mm})$ comparing to the settlement of traditional track (5.3-13 mm) (Figs. 3a \& 5b). Moreover, less lateral dilation is found for the specimen with $R_{b} \geq 10 \%$ (Fig. 4a). Furthermore, the elastic deformation of $1.52 \mathrm{~mm}$ for the specimen with SEAL10 is comparable to a traditional track (Fig. 5b) indicating an acceptable level of vibration. This result also suggests that $R_{b}=10 \%$ is a proper rate to add into SEAL to ensure the track will have acceptable settlement and less lateral dilation than a traditional track without experiencing greater vertical vibration.

\subsection{Energy absorbing property and ballast degradation}

Fig. 6 shows that the hysteretic loop of the track specimen at the end of each test varies according to the amount of rubber added. The hysteretic loop of the traditional track specimen is similar to 
the specimen with SEAL0, albeit with less permanent vertical strain. Note that as more rubber is added to the SEAL matrix, the hysteretic loop of the track specimen expands and shifts to the right, and when $R_{b}$ increases to $40 \%$, the subsequent increase in the area of the hysteretic loop is substantial. This further proves how the addition of rubber increases the permanent and elastic strain. Moreover, this increase in the loop area also indicates a higher dissipated energy. This has been further elaborated through Fig. 7 where the elastic energy density $\left(E_{\text {elastic }}\right)$ and the dissipated energy density $\left(E_{d}\right)$ of the track specimen at the end of each test are presented.

The dissipated energy density can be represented through the area of the hysteretic loop, whereas the elastic energy density refers to the area below the unloading line for each loading cycle (Qi and Indraratna 2020), as shown in Fig. 7. When $R_{b}$ increases from 0 to $20 \%$, both $E_{\text {elastic }}$ and $E_{d}$ increase, albeit this increase in dissipated energy is more pronounced (Fig. 7). However, while the $E_{\text {elastic }}$ and $E_{d}$ of the track specimens having SEAL20 and SEAL30 are similar, the track specimen with SEAL40 experienced a sharp increase in $E_{\text {elastic }}$ and $E_{d}$. This indicates that the skeleton of the SEAL40 matrix is now controlled by the rubber particles which induce a rubberlike behaviour, i.e. a large elastic strain and high compressibility due to larger voids between particles (Indraratna et al. 2020, Tawk et al. 2020). The sum of elastic energy and dissipated energy gives the total amount of absorbed energy by the track substructure. It is therefore easy to conclude that as more rubber is added to SEAL, more energy is absorbed by the track specimen.

While this increase in dissipated energy may result in more energy being consumed by plastic deformation and/or particle breakage (Qi and Indraratna 2020), using SEAL with a higher $R_{b}$ may not be a favourable outcome. To investigate this possibility further, ballast particles directly beneath the sleeper were collected and sieved after each test to obtain the particle size distribution curves, and the ballast breakage index (BBI) was adopted in this study to evaluate ballast breakage 
226 for each test. The value of BBI can be calculated based on the ballast grading curves before and

227 after testing, as initially proposed by Indraratna et al. (2005). The definition of BBI is shown in

228 Fig. 8a, and the BBI obtained after each test is shown in Fig. 8b. Basically, when $10 \%$ rubber is

229 added to the SEAL matrix the BBI decreased by almost $60 \%$, but when more rubber is added to

230 SEAL there is no further improvement, which suggests that $10 \%$ rubber in SEAL is sufficient to

231 mitigate ballast degradation. Note also that the BBI of the traditional track specimen is similar to

232 the test specimen having SEALO.

233 The test results of BBI in Fig. 8b show that the more dissipated energy induced by adding rubber

234 does not result in a higher particle breakage, it actually induces greater plastic deformation, as

235 shown in Fig. 5b. This further indicates that the addition of rubber could reduce ballast breakage

236 by enabling more energy to be consumed by plastic deformation. The ideal percentage of rubber

237 in SEAL is expected to reduce ballast breakage without inducing extensive deformation, in

238 comparison to traditional track materials. Therefore, $10 \%$ rubber is the recommended amount for

239 a SEAL matrix in terms of ballast degradation and deformation.

$240 \quad 2.4$ Track modulus and damping capacity

241 The stiffness and damping properties are the key parameters governing the dynamic performance

242 of rail track (e.g. vibration, deformation and energy dissipation). The track modulus $(K)$ is

243 commonly used to indicate the vertical stiffness of a track supporting system that includes the

244 faster, the sleepers and track substructure (i.e. ballast, subballast and subgrade), that can be

245 calculated by Equations (1-2), as suggested by Selig and Li (1994):

$$
K=\frac{k^{4 / 3}}{(64 E I)^{1 / 3}}
$$


246 where $k$ is the stiffness of the entire track structure which considering the rail bending stiffness $E I$,

247 it can be obtained by:

$$
k=\frac{q}{\delta}
$$

248 where $q$ is the per unit length vertical supporting stress provided by the track component, and $\delta$ is 249 the vertical track deflection. As the track stiffness based on overall vertical deformation is directly 250 influenced by the entire substructure assembly, it is assumed that the calculated value and the 251 proposed relationship for track modulus in this particular study will be suitable for a track 252 substructure that is relatively stiff (e.g. well-compacted ballast interlocked with concrete ties, stiff 253 E-type clip fastener system, solid subgrade) as have been described in the section of Materials and 254 Test Program.

255 Damping refers to the loss of energy within a vibrating or cyclically loaded system. The damping 256 efficiency can be evaluated using the damping ratio (D) which is the ratio of the dissipated energy 257 to the maximum elastic energy stored during one loading cycle. It can be calculated through the 258 hysteretic loop during the cyclic loading, as shown in Fig. 9 b.

259 The track modulus and damping ratio of the track specimen that vary with the loading cycles are 260 shown in Fig. 9(a,b). As the loading cycles evolve, the track modulus increases at the beginning 261 of each test as the test specimens become denser and rapidly stabilise for the remainder of the test. 262 By increasing the amount of rubber in the SEAL matrix the track modulus decreases which is a 263 direct result caused by the increasing vertical strain but with the same the dynamic load amplitude 264 (Fig. 6), and also it is easy to understand because the shear strength of the rubber materials is less 265 than the other two waste materials in the SEAL mixture (i.e. SFS and CW) (Qi et al. 2019a). 266 Compared to traditional track materials, all the test specimens other than that with SEAL0 have a 
267 lower track modulus. A higher track modulus (i.e. track stiffness) always helps to ensure the track

268 has less vibration and deformation, but this may induce a higher interaction force between the

269 sleeper and the ballast due to load concentration (Indraratna et al. 2017). Therefore, it is better for

270 a track foundation with a SEAL matrix (i.e. SEAL10) to have a reasonable comparable track

271 modulus with the traditional track rather than have a much higher or lower value.

272 The track modulus at the end of each test is shown in Fig. 9c after each test; the figure shows there

273 is an exponential relationship between $K$ and $R_{b} \%$ with a high regression coefficient of $R^{2}=$

$274 \quad 0.94:$

$$
K^{*}=\alpha_{1} e^{\alpha_{2}\left(R_{b}+0.1\right)}
$$

275 where $\alpha_{1}$ and $\alpha_{2}$ are the fitting coefficients whose values are shown in Fig. 9c.

276 Note that the test specimen with SEAL0 has a similar damping ratio to the traditional track

277 specimen, but as $R_{b}$ in the SEAL matrix increases the damping ratio also increases, i.e. a higher 278 energy dissipation efficiency (Fig. 9b). This indicates that as more rubber is added, more energy 279 is dissipated through permanent deformation or/and particle breakage rather than in the form of 280 elastic energy. This is also shown in Fig. 7 where the dissipated energy density gradually exceeds 281 the elastic energy density and dominates as $R_{b}$ increases.

282 Track vibration is a complex phenomenon sourced from the moving loads of the train and its 283 propagation depends mainly on the track stiffness and the damping effect of the track substructure.

284 This damping ratio however, is only a relative ratio that reflects the relationship between the 285 dissipated energy and elastic energy rather than directly showing the damping effect of the test 286 specimen. The damping effect of a system that will slow the vibration when subjected to dynamic 287 loading can be evaluated by utilising the viscous damping coefficient (C). This is a theoretical 
288 parameter that can explain how the energy dissipation due to friction can slow the motion of the

289 system under dynamic loading (Escalante-Martínez et al. 2016). The viscous damping coefficient

290 (C) strongly depends on the shear modulus and damping ratio of a system, and it can be obtained

291 by using the following equations:

$$
\begin{gathered}
C=C_{c} \times D \\
C_{c}=2 \sqrt{K m}
\end{gathered}
$$

292 where $C_{c}$ is the critical damping coefficient and $m$ is the unit mass of the material in the system 293 being considered.

294 The viscous damping coefficient (C) for each track specimen obtained at the end of the test is 295 shown in Fig. 9c. Basically, it increases as $R_{b}$ increases to $10 \%$ and then decreases as more rubber 296 is added into the SEAL mixture, which suggests that SEAL with $10 \%$ rubber can act as a damping 297 cushion in the rail foundation to slow the dynamic vibration. Moreover, an empirical relationship 298 between $\mathrm{C}$ and $R_{b} \%$ can be obtained as shown by Equation (6), with a reasonably high coefficient 299 of determination $R^{2}=0.92$.

$$
C^{*}=\beta_{1}\left(R_{b}\right)^{2}+\beta_{2} R_{b}+\beta_{3}
$$

300 where $\beta_{1,2,3}$ are the fitting coefficients whose values are shown in Fig. 9c.

\section{$301 \quad 2.5$ Dynamic amplification factor}

302 Under dynamic loading conditions, the actual stress imparted by the track foundation is usually 303 higher than the applied load. One of the main functions of the subballast layer is to distribute the 304 load and reduce the stress being transmitted to other layers. To investigate how the incorporation 305 of SEAL will influence the interface stress between each layer of track substructure, the measured 
stress at the interface of each test specimen is shown in Fig. 10a. Note here that the measured stress

307 at the interface decreases along the depth of the test specimen. At the interface of the same layer,

308 the track specimen with SEAL10 has the lowest stress while the specimen with SEAL40 has the

309 highest, and the stress at the interface of the traditional track specimen is higher than the specimen

310 with SEAL10 but lower than the other test specimens.

311 The dynamic amplification factor (DAF) is used to evaluate the dynamic loading in this study. It

312 is a dimensionless parameter. It is the ratio between the maximum stress caused by the dynamic

313 or cyclic load to the maximum deviator stress applied to the structure, and can be obtained via

314 Equation (7), as suggested by Sun et al. (2016):

$$
\mathrm{DAF}=q_{d, \max } / q_{\max , c y c}
$$

315 where $q_{d, \max }$ is the peak dynamic deviator stress measured during the cyclic loading test, and $316 q_{\max , c y c}$ is the applied maximum deviator stress.

317 The DAF of the test specimen that varies with the amount of rubber is shown in Fig. 10b. This 318 figure shows that DAF decreases from the top layer of ballast to the bottom layer of subgrade as 319 the stress is distributed alongside the depth. When $10 \% \mathrm{RC}$ is added in the SEAL matrix, DAF 320 decreases slightly from 1.25 to 1.1 and then increases as $R_{b}$ increases. Note that the DAF on top 321 of ballast of the specimen with SEAL40 is more than double that of the specimen with SEAL10, 322 and the interface stress on top of the ballast has doubled compared to the pressure applied due to 323 the dynamic effect. The additional stress generated under the dynamic environment depends 324 mainly on the lateral confinement, the loading frequency, the track stiffness and damping effect, 325 and the energy absorbing capacity of the track substructure (Esveld and Esveld 2001). A track 326 substructure with a relatively high track stiffness, low damping coefficient, and low energy 
327 absorbing capacity that is subjected to a high lateral confining pressure and high loading frequency

328 will generate a high DAF, and this extensive additional stress may result in high deformation and

329 ballast breakage. Given that the lateral confinement and loading frequency are controlled the same

330 during testing, the DAF for the track specimen in this study is influenced by a combination of track

331 stiffness, damping coefficient, and energy absorbing capacity. Furthermore, since the track

332 specimen with SEAL10 has the lowest DAF, it is recommended that the optimal percentage of

333 rubber should be $10 \%$ in a SEAL matrix when the dynamic amplification effect is considered.

3. Predicted dynamic response of rail with SEAL incorporated track

335 To investigate how SEAL will affect the dynamic response (vertical displacement and acceleration)

336 of rail in field conditions, a simple track dynamic model considering a platoon of moving line

337 loads is adopted in this study (Fig. 11). The rail is considered to be a Bernoulli-Euler beam resting

338 on a viscoelastic foundation that incorporates SEAL. This viscoelastic foundation is equivalent to

339 a spring and dashpot system (Fig. 11) as the track foundation is simplified as a complete system

340 during prismoidal triaxial testing to measure the track modulus and viscous damping coefficient.

341 Assuming the rail deflection is $u$ in the vertical direction, the moving load is travelling in $x$

342 direction (horizontally along the track), and the time is $t$, the origin of the coordinate system is set

343 at the middle of the distribution of the last moving load. The common governing equation of a

344 Bernoulli-Euler beam on a viscoelastic foundation is:

$$
E I u^{(4)}(x)+K^{*} u+C^{*} \dot{u}(t)+m \ddot{u}(t)=F(x, t)
$$

345 where $E I$ is the bending stiffness of the rail, $K^{*}$ and $C^{*}$ are the stiffness and the viscous damping 346 coefficient of the track substructure that varies with the percentage of rubber in SEAL, as denoted 
347 by Equations (3) and (6), respectively. Assuming the length of the rail is infinite, the boundary

348 conditions are: $u( \pm \infty)=0 ; \lim _{x \rightarrow \pm \infty} u^{(j)}\left(x^{j}\right)=0, j=1,2,3,4$.

$349 F(x, t)$ is the external dynamic load (a platoon of uniform moving line loads with uniform 350 distributions), as represented by Equation (9) (Sun and Luo 2008):

$$
F(x, t)=\sum_{n=1}^{n} P \exp (i \Omega t)(2 r)^{-1} H\left(r-\left|x-v t-\sum_{n=1}^{n} l_{n}\right|\right)
$$

351 where $i$ is a unit imaginary number. Each load is $2 r$ long and $n$ is the number of moving loads, 352 and $P$ and $\Omega$ are the amplitude and frequency of the jth load, respectively. $l$ is the space between 353 the middle point of two adjacent moving loads. The moving load is travelling at a speed of $v$, and $354 H(\cdot)$ is the unit Heaviside step function defined as:

$$
H\left(x-x_{0}\right)=\left\{\begin{array}{l}
0 \text { for } x<x_{0} \\
\frac{1}{2} \text { for } x=x_{0} \\
1 \text { for } x>x_{0}
\end{array}\right.
$$

355 Applying the Fourier transform to Equation (8) and rearranging it gives:

$$
\tilde{u}(\xi, \omega)=\frac{\tilde{F}(\xi, \omega)}{E I \xi^{4}+K^{*}+i C^{*} \omega-m \omega^{2}}
$$

356 Applying the inverse Fourier transform to Equation (11) gives an integral representation of the 357 steady-state dynamic displacement of the beam in the time domain:

$$
u(x, t)=(2 \pi)^{-2} \int_{-\infty}^{\infty} \int_{-\infty}^{\infty} \frac{\tilde{F}(\xi, \omega) \exp [i(\xi x+\omega t)]}{E I \xi^{4}+K^{*}+i C^{*} \omega-m \omega^{2}} d \xi d \omega
$$

358 Applying the Fourier transform to Equation (9): 


$$
\begin{aligned}
\tilde{F}(\xi, \omega)= & \int_{-\infty}^{\infty} \int_{-\infty}^{\infty} \sum_{n=1}^{n} P \exp (i \Omega t)(2 r)^{-1} H\left(r-\left|x-v t-\sum_{n=1}^{n} l_{n}\right|\right) \exp [-i(\xi x \\
& +\omega t)] d x d t
\end{aligned}
$$

359 Note that:

$$
\begin{aligned}
\int_{-\infty}^{\infty}(2 r)^{-1} H(r & \left.-\left|x-v t-\sum_{n=1}^{n} l_{n}\right|\right) \exp [-i(\xi x) d x \\
& =\int_{v t+\sum_{n=1}^{n} l_{n}-r_{0}}^{v t+\sum_{n=1}^{n} l_{n}+r_{0}} \frac{\exp (-i \xi x)}{2 r_{0}} d x \\
& =\frac{\sin r \xi}{r \xi} \exp \left[-i \xi\left(v t+\sum_{n=1}^{n} l_{n}\right)\right]
\end{aligned}
$$

360

$$
\int_{-\infty}^{\infty} \exp [-i(\omega+v \xi-\Omega) t] d t=2 \pi \delta(\omega+v \xi-\Omega)
$$

361 where $\delta(x)$ is the Dirac function.

362 Substituting Equations (14-15) into Equation (13) gives:

$$
\tilde{F}(\xi, \omega)=\sum_{n=1}^{n} 2 \pi P \exp \left(-i \xi \sum_{n=1}^{n} l_{n}\right)(r \xi)^{-1} \sin (r \xi)(\omega+v \xi-\Omega)
$$

363 Substituting Equation (16) into Equation (12) gives the vertical displacement of the rail: 


$$
\begin{aligned}
u(x, t)=\sum_{j=1}^{J} \frac{\bar{P}}{2 \pi} & \exp (i \Omega t) \\
& \times \int_{-\infty}^{\infty} \frac{E I \sin (r \xi) \exp [i \xi(x-v t)] \exp \left(-i \xi \sum_{n=1}^{n} l_{n}\right)}{r \xi\left[E I \xi^{4}+K^{*}+i C^{*}(\Omega-v \xi)-m \omega(\Omega-v \xi)^{2}\right)} d \xi
\end{aligned}
$$

364 The vertical acceleration of the beam can then be obtained by differentiating Equation (17):

$$
\begin{array}{ll}
\text { Acceleration: } & \ddot{u}_{t}(x, t) \\
& =\sum_{n=1}^{n} \frac{\bar{P}}{2 \pi} \exp (i \Omega t) \\
& \times \int_{-\infty}^{\infty} \frac{E I(\Omega-v \xi)^{2} \sin (r \xi) \exp [i \xi(x-v t)] \exp \left(-i \xi \sum_{n=1}^{n} l_{n}\right)}{r \xi\left[E I \xi^{4}+K^{*}+i C^{*}(\Omega-v \xi)-m \omega(\Omega-v \xi)^{2}\right)} d \xi
\end{array}
$$

365 All the parameters used for numerical computing this track dynamic model are listed in Table 1.

366 The listed loading condition and the parameters of the track foundation simulate field loading conditions. $\mathrm{P}=50 \mathrm{kN}$ and $\Omega=15 \mathrm{~Hz}$ are the amplitude of the load and the loading frequency, respectively, used to simulate a train with a 25 -tonne axle load running at $115 \mathrm{~km} / \mathrm{h}$. The values of

$369 \mathrm{~K}$ and $\mathrm{C}$ for each track having different SEAL matrices are obtained from the large scale prismoidal triaxial tests. The length of the load distribution is $2 r=15.75 \mathrm{~m}$ and the space between the two

371 middle points of two adjacent loads is assumed to be $l=18.75 \mathrm{~m}$; this simulates standard 372 suburban carriage stock in New South Wales, Australia (Punetha et al. 2020).

373 The dynamic responses (i.e. displacement and acceleration) of a rail subjected to a single moving 374 load with varying viscous damping coefficients and track stiffness in the track substructure are 375 shown in Fig. 12 and Fig. 13, respectively. The observation point is at $x=0$, which is the point of 376 origin of the coordinate system, and where $t=0$ means the moving load is passing through the 
377 observation point. Both negative and positive values can be observed for the rail dynamic response,

378 indicating the rail experiences tensile and compressive stresses as the moving load passes by.

379 When the magnitude of the viscous damping coefficient is changed while all the other parameters

380 are the same, there is a marginal change in displacement and acceleration as $\mathrm{C}$ increases from $10^{4}$

381 to $10^{5} \mathrm{Ns} / \mathrm{m}^{2}$, and a large reduction (50-80\%) when C increases from $10^{5}$ to $10^{6} \mathrm{Ns} / \mathrm{m}^{2}$ and

382 then to $10^{7} \mathrm{Ns} / \mathrm{m}^{2}$. Moreover, peak displacement and acceleration are not sensitive to changes in

383 the track stiffness from $10^{5}$ to $10^{6} \mathrm{~N} / \mathrm{m}^{2}$ but they decrease significantly when the track stiffness

384 varies between $10^{6}$ to $10^{9} \mathrm{~N} / \mathrm{m}^{2}$. Furthermore, a reduction in track stiffness induces vertical

385 deflection to takes longer to recover (Fig. 13a). Given that the track stiffness and viscous damping

386 coefficient of track substructure will generally vary between $10^{5} \leq C \leq 10^{7} \mathrm{~N} / \mathrm{m}^{2}$ and $10^{6} \leq$

$387 K \leq 10^{9} \mathrm{~N} / \mathrm{m}^{2}$, the damping property and track stiffness of the track substructure are the two key

388 factors that influence rail deformation and vibration.

389 Because changing the amount of rubber in the subballast layer changes the damping property and 390 track stiffness, a plot of the dynamic response of rail that varies with the amount of rubber shows 391 a combination change of the damping property and track stiffness, as shown in Fig. 14. The model 392 prediction shows that when increasing the amount of rubber in SEAL, a rail experiences higher 393 displacement and acceleration, which indicates that more rubber can generate more deformation 394 and vibration for the rail track. This agrees with the laboratory test results where more rubber 395 generates more vertical deformation (shown earlier in Fig. 3) and more elastic energy (see Fig. 7).

396 Moreover, when the percentage of rubber in SEAL increases to $40 \%$ the subsequently sharp jump 397 in displacement and acceleration is almost 2-3 times greater than without rubber. This matches the 398 laboratory observation that the track with SEAL40 collapsed with severe vibration and 

or even less vertical deflection and acceleration.

401 To obtain a further space-time overview of how the percentage of rubber in SEAL affects the 402 vertical vibration of rail, a three-dimensional view of the acceleration of rail by applying a platoon 403 of ten moving loads (i.e. $n=10$ ) is shown in Fig. 15. The peak acceleration occurs almost at the 404 point of origin of the coordinate system. It is noted that the amplitude of acceleration increases as 405 more rubber is added to the track foundation. The peak acceleration of track with SEAL40 is 406 almost $60 \%$ greater than with SEAL0, whereas acceleration with SEAL10 is comparable to a 407 traditional track, and this is in line with the laboratory observations.

408 Overall, the track dynamic model further validates how the percentage of rubber crumbs used in 409 the subballast layer will affect the dynamic response of track, such that more rubber generates 410 more settlement and vibration, while the settlement and vibration of the track with SEAL10 is 411 comparable to traditional track. Furthermore, utilising SEAL10 leads to less lateral dilation, less 412 ballast degradation, and less dynamic pressure at the interface of the substructure. Therefore, $10 \%$ 413 rubber is recommended to be included in SEAL to replace traditional subballast materials.

\section{Limitations of the study}

415 Apart from the properties of the substructure materials, track dynamic response is also influenced 416 by other parameters such as the excitation frequency, varied axle loads from different rolling stock 417 generating a wider array of cyclic stress ratios as well as the structural assembly of track and the 418 type of gauge (i.e. geometry and ballast-sleeper assemblies). The individual roles of all these 419 influential factors could not be considered within the scope of this single study. Consequently, the 420 contents of this paper are subjected to certain limitations within its current scope in relation to the 421 following simplifications and assumptions. 
422 (i) The computed track modulus is based on the assumption of a relatively stiff track substructure 423 consisting of well-compacted ballast and sub-ballast overlying a solid subgrade; the analysis may

424 deviate from accuracy if the substructure layers are considerably softer or compressible.

425 (ii) The laboratory investigation and the track dynamic model were specifically focused on 426 examining the influence of rubber contents in the energy absorbing mixtures on the track dynamic

427 response, while all other contributory factors (e.g. track geometry and structure, loading conditions,

428 ballast and subgrade characteristics) were kept unchanged. Indeed, the track response will be 429 different if other parameters are also varied, for instance if the track construction materials were 430 to be changed.

431 (iii) Only one value of loading frequency $(15 \mathrm{~Hz})$ was used in the current study that would 432 corroborate with the range of $80-110 \mathrm{~km} / \mathrm{h}$ speeds for heavy haul trains in Australia depending on 433 the type of track gauge and the bogey spacing of freight trains. Naturally, either much smaller or 434 much greater frequencies for the same applied cyclic load will generate varied track dynamics.

\section{Conclusions}

436 In this paper, a synthetic energy absorbing layer (SEAL) was proposed to replace traditional 437 subballast material. The performance of track specimens with SEAL was examined through large438 scale prismoidal triaxial tests under cyclic loading by changing the amount of rubber in the SEAL 439 matrix. It was revealed that the amount of RC within the SEAL matrix had a significant influence 440 on the dynamic response (deformation, ballast degradation, track modulus, damping ratio, 441 vibration, stress distribution, and energy absorbing capacity) of the rail foundation. On this basis 442 a track dynamic model was developed to better investigate track performance when SEAL was 443 used. The following conclusions can be drawn from this study: 
(1) When the amount of rubber in the SEAL matrix was increased the permanent and elastic vertical displacement of the track specimen increased, but its lateral dilation decreased. When the rubber content in SEAL was $\geq 20 \%$, lateral deformation fluctuated as compression and dilation appeared alternately. The test specimen with SEAL40 reached plastic collapse with extensive settlement and excessive vibration at around 1500 loading cycles, whereas all the other specimens achieved plastic shakedown before the test ended.

(2) The addition of rubber in SEAL increased the total energy absorbing capacity of the track specimen and mitigated ballast breakage. When $10 \%$ of rubber was added to SEAL, the

BBI decreased by almost $60 \%$, but when more rubber was added there was no further benefit. This implies that for each type and gradation of granular material, an optimum rubber content exists beyond which the returns are marginal.

(3) Adding resilient rubber to the SEAL matrix reduced the track modulus and increased the damping ratio of the track specimen (i.e. the efficiency to dissipate energy). The viscous damping coefficient which reflected its ability to reduce motion increased as $10 \%$ rubber was included, after which it decreased again. These results imply that the damping coefficient of the SEAL is directly related to the optimum rubber content. While it was evaluated at $10 \%$ for the material tested herein, for significantly different types of granular soils and rockfills the optimum rubber content may deviate from $10 \%$.

(4) The pressure at the interface and DAF decreased along with the depth of the track foundation. The test specimen with SEAL10 had the lowest pressure and DAF at the interface of each layer, whereas the interface pressure and DAF increased as rubber contents increased beyond $10 \%$. 
(5) A dynamic track model was developed to investigate the dynamic response of rail tracks incorporating the SEAL matrix. It was found that the dynamic response of rail (deflection and acceleration) was mainly affected by the track modulus and damping property, both of which were governed by changing the percentage of rubber in SEAL. While the addition of rubber increased the vertical deflection and acceleration of the track, the use of SEAL10 would still ensure an acceptable dynamic response.

472

(6) Compared to the traditional track specimens, adding 10\% rubber in SEAL reduced lateral dilation, ballast degradation, and stresses developed at the interface, while maintaining an acceptable level of vertical deformation and vibration. On this basis SEAL10 can be recommended to be a promising option for rubber-blended capping or subballast materials to replace traditional subballast in rail tracks. 
479 Some or all data, models, or code that support the findings of this study are available from the 480 corresponding author upon reasonable request.

\section{Acknowledgements}

482 The authors would like to acknowledge the financial support provided by the Australian Research 483 Council Discovery Project (ARC-DP; project ID: DP180101916). The assistance provided by 484 industry (ASMS, South 32, and Tire Crumb Australia) in relation to the procurement of material 485 used in this study is gratefully acknowledged.

\section{References}

487 Escalante-Martínez, J., Gómez-Aguilar, J., Calderón-Ramón, C., Morales-Mendoza, L., Cruz488 Orduna, I. and Laguna-Camacho, J. (2016). "Experimental evaluation of viscous damping 489 coefficient in the fractional underdamped oscillator." Advances in Mechanical Engineering 8(4): 4901687814016643068.

491 Esmaeili, M., Aela, P. and Hosseini, A. (2017). "Experimental assessment of cyclic behavior of 492 sand-fouled ballast mixed with tire derived aggregates." Soil Dynamics and Earthquake 493 Engineering 98: 1-11.

494 Esveld, C. and Esveld, C. (2001). Modern railway track, MRT-productions Zaltbommel, 495 Netherlands.

496 Fathali, M., Esmaeili, M. and Nejad, F. M. (2019). "Influence of tire-derived aggregates mixed 497 with ballast on ground-borne vibrations." Journal of Modern Transportation 27(4): 355-363.

498 Fernandes, G., Palmeira, E. M. and Gomes, R. C. (2008). "Performance of geosynthetic-reinforced 499 alternative sub-ballast material in a railway track." Geosynthetics International 15(5): 311-321. 
500 Fernández, P. M., Signes, C. H., Sanchís, I. V., Mira, D. P. and Franco, R. I. (2018). "Real scale 501 evaluation of vibration mitigation of sub-ballast layers with added tyre-derived aggregate." 502 Construction and Building Materials 169: 335-346.

503 Gong, H., Song, W., Huang, B., Shu, X., Han, B., Wu, H. and Zou, J. (2019). "Direct shear 504 properties of railway ballast mixed with tire derived aggregates: Experimental and numerical 505 investigations." Construction and Building Materials 200: 465-473.

506 Indraratna, B., Ferreira, F. B., Qi, Y. and Ngo, T. N. (2018a). "Application of geoinclusions for 507 sustainable rail infrastructure under increased axle loads and higher speeds." Innovative 508 Infrastructure Solutions 3(1): 69.

509 Indraratna, B., Lackenby, J. and Christie, D. (2005). "Effect of confining pressure on the 510 degradation of ballast under cyclic loading." Geotechnique 55(4): 325-328.

511 Indraratna, B. and Nimbalkar, S. (2013). "Stress-strain degradation response of railway ballast 512 stabilized with geosynthetics." Journal of geotechnical and geoenvironmental engineering 139(5): $513 \quad 684-700$.

514 Indraratna, B., Qi, Y. and Heitor, A. (2018b). "Evaluating the properties of mixtures of steel 515 furnace slag, coal wash, and rubber crumbs used as subballast." Journal of Materials in Civil 516 Engineering 30(1): 04017251.

517 Indraratna, B., Qi, Y., Ngo, T. N., Rujikiatkamjorn, C., Neville, T., Ferreira, F. B. and Shahkolahi, 518 A. (2019). "Use of geogrids and recycled rubber in railroad infrastructure for enhanced 519 performance." Geosciences 9(1): 30.

520 Indraratna, B., Qi, Y., Tawk, M., Heitor, A., Rujikiatkamjorn, C. and Navaratnarajah, S. K. (2020). 521 "Advances in Ground Improvement Using Waste Materials for Transportation Infrastructure." 522 Proceedings of the Institution of Civil Engineers-Ground Improvement: 1-44. 
523 Indraratna, B., Sun, Q. and Grant, J. (2017). "Behaviour of subballast reinforced with used tyre 524 and potential application in rail tracks." Transportation Geotechnics 12: 26-36.

525 Indraratna, B., Sun, Q., Heitor, A. and Grant, J. (2018c). "Performance of rubber tire-confined 526 capping layer under cyclic loading for railroad conditions." Journal of Materials in Civil 527 Engineering 30(3): 06017021.

528 Jayasuriya, C., Indraratna, B. and Ngo, T. N. (2019). "Experimental study to examine the role of 529 under sleeper pads for improved performance of ballast under cyclic loading." Transportation 530 Geotechnics 19: 61-73.

531 Kaewunruen, S., Aikawa, A. and Remennikov, A. M. (2017). "Vibration attenuation at rail joints 532 through under sleeper pads." Procedia engineering 189: 193-198.

533 Kaewunruen, S. and Remennikov, A. M. (2016). "Current state of practice in railway track 534 vibration isolation: an Australian overview." Australian Journal of Civil Engineering 14(1): 6353571.

536 Lackenby, J., Indraratna, B., McDowell, G. and Christie, D. (2007). "Effect of confining pressure 537 on ballast degradation and deformation under cyclic triaxial loading." Géotechnique 57(6): 527538536.

539 Lakušić, S., Ahac, M. and Haladin, I. (2010). Experimental investigation of railway track with 540 under sleeper pad. 10th Slovenian road and transportation congress.

541 Li, D. and Selig, E. T. (1995). "Wheel/track dynamic interaction: track substructure perspective." $542 \quad$ Vehicle System Dynamics 24(sup1): 183-196.

543 Navaratnarajah, S. K. and Indraratna, B. (2017). "Use of rubber mats to improve the deformation 544 and degradation behavior of rail ballast under cyclic loading." Journal of geotechnical and 545 geoenvironmental engineering 143(6): 04017015. 
546 Navaratnarajah, S. K., Indraratna, B. and Ngo, N. T. (2018). "Influence of under sleeper pads on

547 ballast behavior under cyclic loading: experimental and numerical studies." Journal of

548 Geotechnical and Geoenvironmental Engineering 144(9): 04018068.

549 Ngo, T. N., Indraratna, B. and Rujikiatkamjorn, C. (2019). "Improved performance of ballasted 550 tracks under impact loading by recycled rubber mats." Transportation Geotechnics 20: 100239.

551 Nimbalkar, S., Indraratna, B., Dash, S. K. and Christie, D. (2012). "Improved performance of 552 railway ballast under impact loads using shock mats." Journal of geotechnical and 553 geoenvironmental engineering 138(3): 281-294.

554 Punetha, P., Nimbalkar, S. and Khabbaz, H. (2020). "Analytical Evaluation of Ballasted Track 555 Substructure Response under Repeated Train Loads." International Journal of Geomechanics 556 20(7): 04020093.

557 Qi, Y. and Indraratna, B. (2020). "Energy-Based Approach to Assess the Performance of a 558 Granular Matrix Consisting of Recycled Rubber, Steel-Furnace Slag, and Coal Wash." Journal of $559 \quad$ Materials in Civil Engineering 32(7): 04020169.

560 Qi, Y., Indraratna, B. and Coop, M. R. (2019a). "Predicted behavior of saturated granular waste 561 blended with rubber crumbs." International Journal of Geomechanics 19(8): 04019079.

562 Qi, Y., Indraratna, B., Heitor, A. and Vinod, J. S. (2018a). "Effect of rubber crumbs on the cyclic 563 behavior of steel furnace slag and coal wash mixtures." Journal of Geotechnical and 564 Geoenvironmental Engineering 144(2): 04017107.

565 Qi, Y., Indraratna, B., Heitor, A. and Vinod, J. S. (2019b). "Closure to "Effect of Rubber Crumbs 566 on the Cyclic Behavior of Steel Furnace Slag and Coal Wash Mixtures" by Yujie Qi, Buddhima 567 Indraratna, Ana Heitor, and Jayan S. Vinod." Journal of Geotechnical and Geoenvironmental $568 \quad$ Engineering 145(1): 07018035. 
569 Qi, Y., Indraratna, B. and Vinod, J. S. (2018b). "Behavior of steel furnace slag, coal wash, and 570 rubber crumb mixtures with special relevance to stress-dilatancy relation." Journal of Materials

571 in Civil Engineering 30(11): 04018276.

572 Qi, Y., Indraratna, B. and Vinod, J. S. (2018c). Dynamic Properties of Mixtures of Waste Materials. 573 GeoShanghai International Conference, Springer.

574 Selig, E. T. and Li, D. (1994). "Track modulus: Its meaning and factors influencing it." 575 Transportation Research Record(1470).

576 Sol-Sánchez, M., Moreno-Navarro, F. and Rubio-Gámez, M. C. (2014). "Viability of using end577 of-life tire pads as under sleeper pads in railway." Construction and Building Materials 64: 150578156.

579 Sol-Sánchez, M., Thom, N., Moreno-Navarro, F., Rubio-Gamez, M. and Airey, G. (2015). "A 580 study into the use of crumb rubber in railway ballast." Construction and Building Materials 75: $581 \quad 19-24$.

582 Sun, L. and Luo, F. (2008). "Steady-state dynamic response of a Bernoulli-Euler beam on a 583 viscoelastic foundation subject to a platoon of moving dynamic loads." Journal of Vibration and 584 Acoustics 130(5): 051002.

585 Sun, Q. D., Indraratna, B. and Nimbalkar, S. (2016). "Deformation and degradation mechanisms 586 of railway ballast under high frequency cyclic loading." Journal of Geotechnical and 587 Geoenvironmental Engineering 142(1): 04015056.

588 Tatsuoka, F., Tateyama, M., Koseki, J. and Yonezawa, T. (2014). "Geosynthetic-reinforced soil 589 structures for railways in Japan." Transportation Infrastructure Geotechnology 1(1): 3-53. 
590 Tawk, M., Qi, Y., Indraratna, B., Rujikiatkamjorn, C. and Heitor, A. (2020). "Behaviour of a 591 Mixture of Coal Wash and Rubber Crumbs under Cyclic Loading." Journal of Materials in Civil 592 Engineering.

593 Toward, M. G., Jiang, J., Dijckmans, A., Coulier, P., Thompson, D. J., Degrande, G., Lombaert, 594 G. and Hussein, M. F. (2014). Mitigation of railway induced vibrations by using subgrade 595 stiffening and wave impeding blocks. 9th International Conference on Structural Dynamics 596 (EURODYN 2014).

597 Xin, T. and Gao, L. (2011). "Reducing slab track vibration into bridge using elastic materials in 598 high speed railway." Journal of Sound and Vibration 330(10): 2237-2248.

599 Youwai, S. and Bergado, D. T. (2003). "Strength and deformation characteristics of shredded 600 rubber tire sand mixtures." Canadian geotechnical journal 40(2): 254-264.

601

602

603 


\section{Table list}

605

Table 1 Parameters for SEAL incorporated track

\begin{tabular}{|c|c|c|c|c|c|c|}
\hline Parameters & SEAL 0 & SEAL10 & SEAL20 & SEAL30 & SEAL40 & Traditional \\
\hline $\mathrm{P}(\mathrm{kN})$ & 50 & 50 & 50 & 50 & 50 & 50 \\
\hline $\mathrm{EI}\left(\mathrm{N} / \mathrm{m}^{2}\right)$ & 2300 & 2300 & 2300 & 2300 & 2300 & 2300 \\
\hline $\mathrm{K}\left(\mathrm{N} / \mathrm{m}^{2}\right)$ & $10.7 * 10^{7}$ & $6.3 * 10^{7}$ & $4.8^{*} 10^{7}$ & $4.4^{*} 10^{7}$ & $1.6^{*} 10^{7}$ & $7.1^{*} 10^{7}$ \\
\hline $\mathrm{m}(\mathrm{kg} / \mathrm{m})$ & 2033.58 & 1999.75 & 1974.08 & 1953.41 & 1935.92 & 2011.75 \\
\hline $\mathrm{C}\left(\mathrm{Ns} / \mathrm{m}^{2}\right)$ & $3.46^{*} 10^{5}$ & $4.55^{*} 10^{5}$ & $3.89 * 10^{5}$ & $3.59 * 10^{5}$ & $2.32^{*} 10^{5}$ & $3.03 * 10^{5}$ \\
\hline $\boldsymbol{\Omega}(\mathrm{Hz})$ & 15 & 15 & 15 & 15 & 15 & 15 \\
\hline
\end{tabular}

606

607

608

609 


\section{Figure captions}

611 Fig. 1 Grading curves for ballast, traditional subballast, structural fill, steel furnace slag, coal 612 wash, rubber crumbs, and the SEAL matrix

613 Fig. 2 (a) Large-scale prismoidal triaxial apparatus with a well-prepared specimen; (b) cross614 section view of the 3-layered physical model; (c) cyclic loading conditions

615 Fig. 3 Deformation responses of the track specimens with different SEAL matrix or traditional 616 materials: (a) vertical displacement, (b) accumulation rate of vertical strain

617 Fig. 4 (a) Lateral displacement of the track specimens with different SEAL matrix and traditional 618 materials; and accumulation rate of lateral strain of track specimens with (b-1, b-2) SEAL0-

619 SEAL30 and traditional materials, (b-3) SEAL40

620 Fig. 5 Deformation responses of the track specimens with different SEAL matrix or traditional 621 materials: (a) elastic vertical deformation, and (b) total plastic and elastic deformation

622 Fig. 6 Hysteretic loops of the track specimen with traditional subballast or SEAL matrix having 623 different rubber contents at the end of each test

624 Fig. 7 Energy density of the track specimen with SEAL matrix having different rubber contents 625 at the end of each test

626 Fig. 8 (a) Definition of ballast breakage index (BBI); (b) BBI obtained from each test

627 Fig. 9 Track modulus and damping ratio of the track specimen with SEAL matrix or traditional 628 subballast materials (a-b) changing with loading cycles, and (c) at the end of each test

629 Fig. 10 (a) Measured pressure on top of each layer (i.e. ballast, subballast and structural fill) for 630 each test; (b) DAF for track specimen changing with RC contents within SEAL matrix

631 Fig. 11 A rail track subjected to a platoon of uniform moving line loads

632 Fig. 12 Dynamic response of rail with changing viscous damping coefficients: (a) displacement 633 and (b) acceleration

634 Fig. 13 Dynamic response of rail with changing shear stiffness: (a) displacement and (b) 635 acceleration

636 Fig. 14 Predicted rail dynamic response for traditional track and track incorporated with SEAL 637 (a) displacement and (b) acceleration

638 Fig. 15 Predicted 3-D view of the acceleration for the track with (a) SEAL0, (b) SEAL10, (c)

639 SEAL20, (d) SEAL30 and (e) SEAL40 and (f) traditional materials 


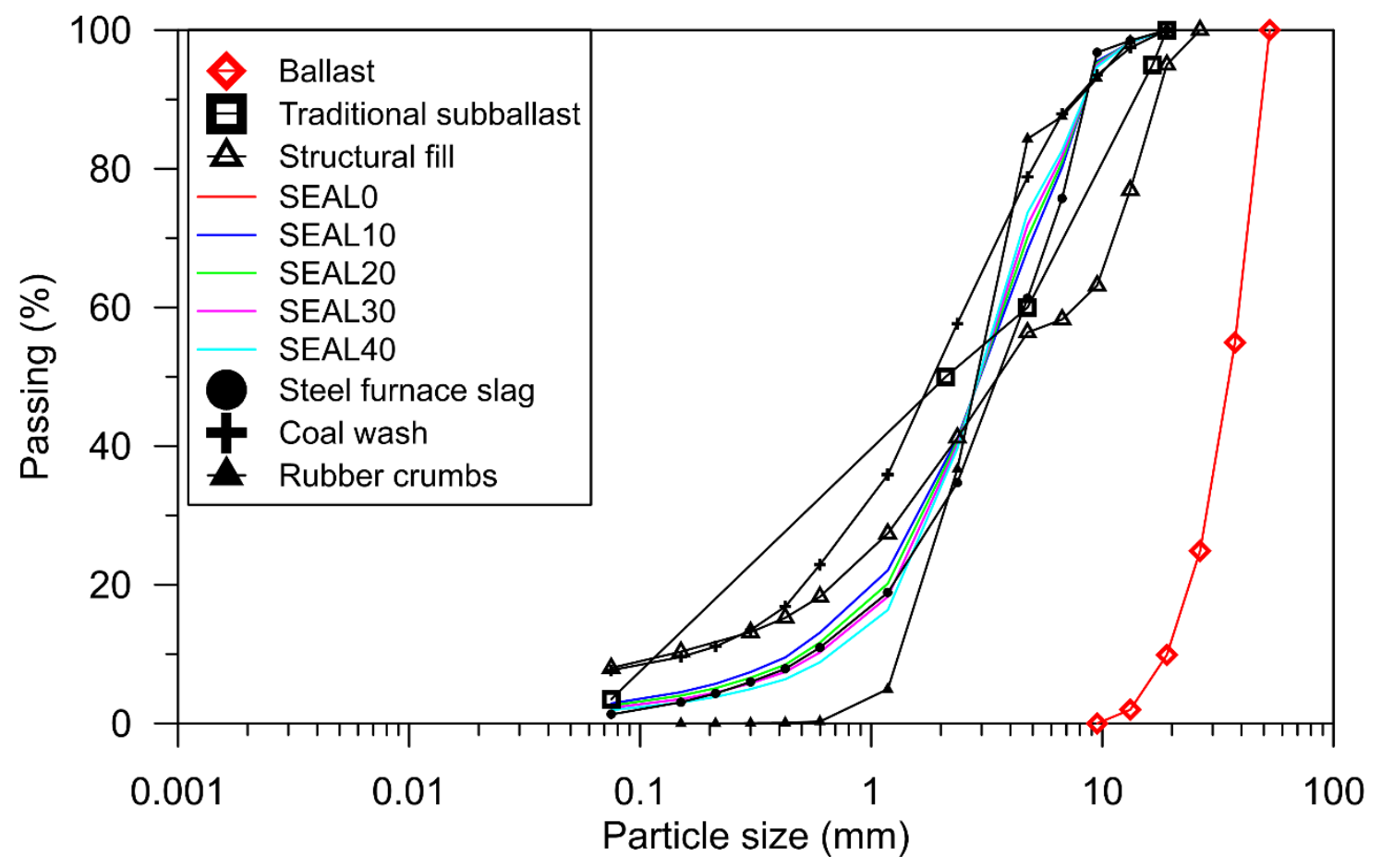

643 Fig. 1 Grading curves for ballast, traditional subballast, structural fill, steel furnace slag, coal 644 wash, rubber crumbs, and the SEAL matrix 


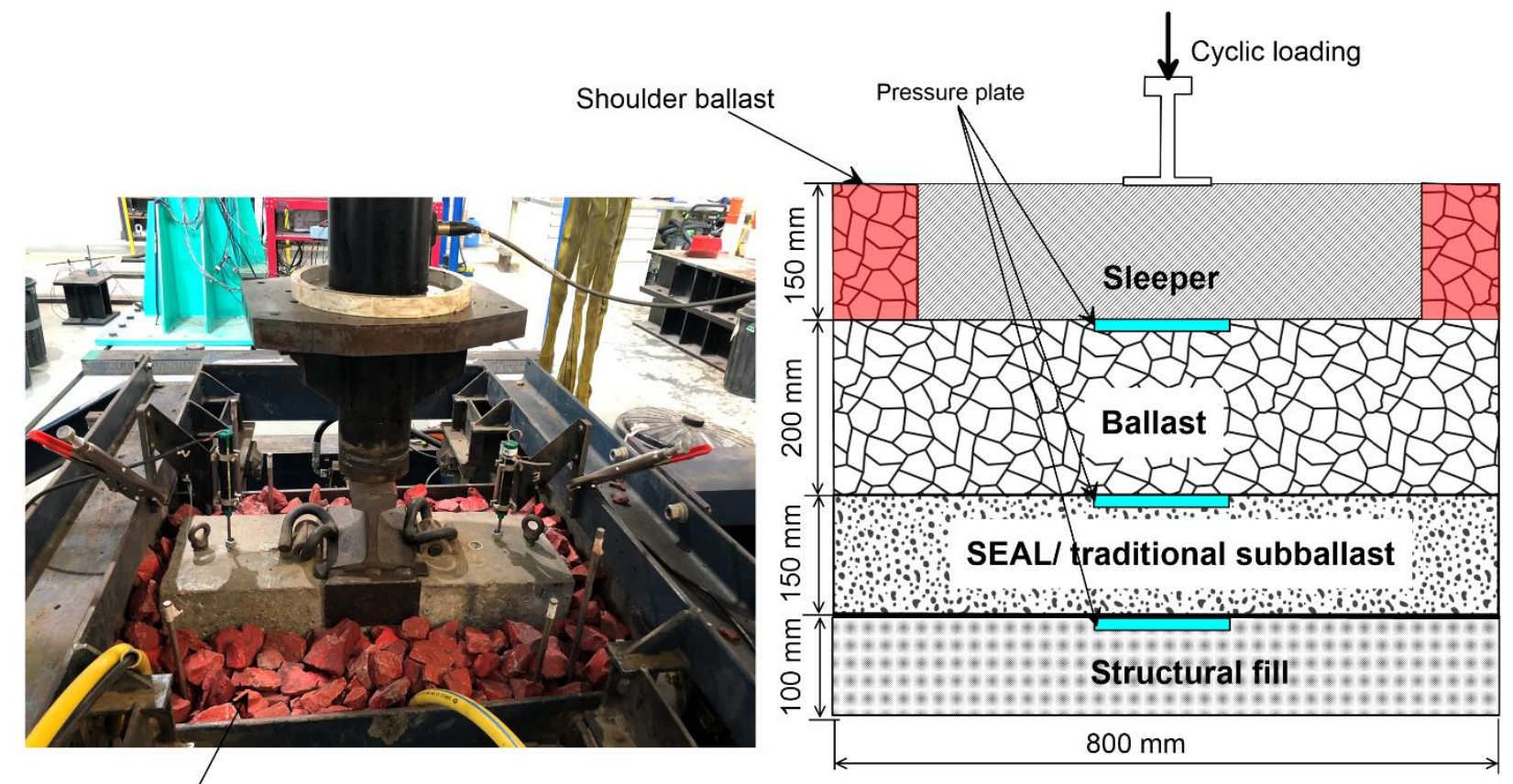

Shoulder ballast

(a)

(b)

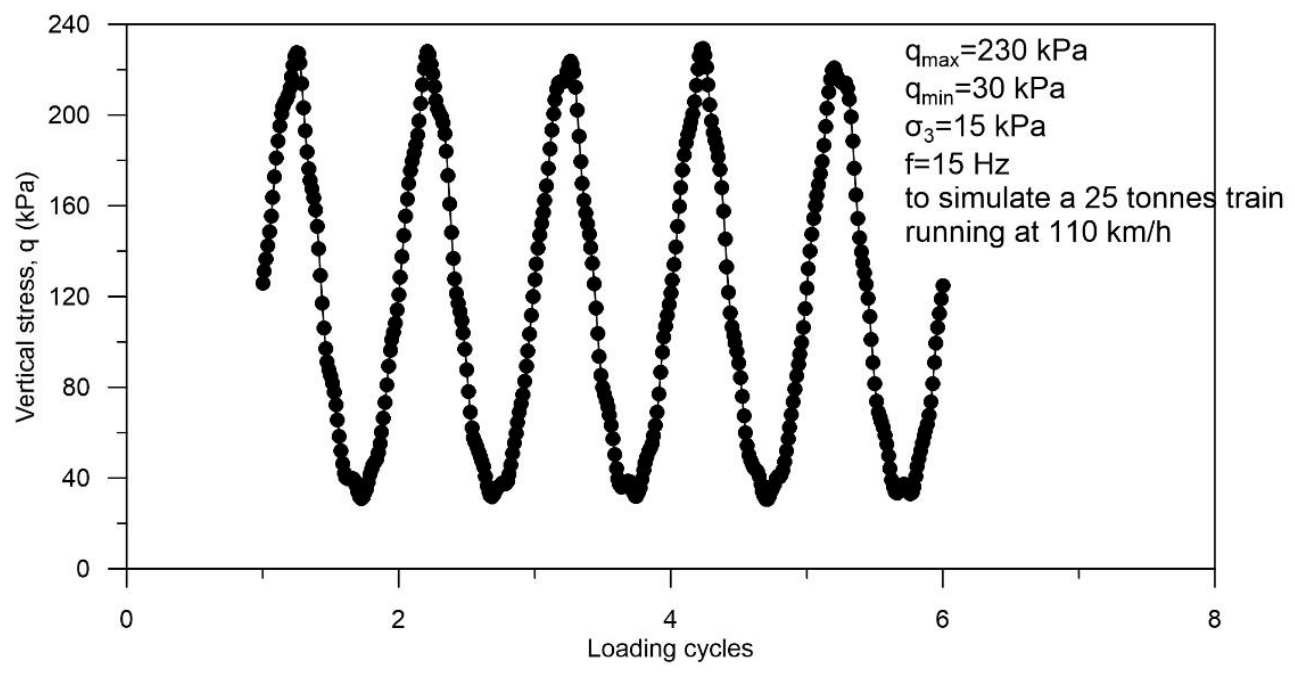

(c)

646 Fig. 2 (a) Large-scale prismoidal triaxial apparatus with a well-prepared specimen; (b) cross647 section view of the 3-layered physical model; (c) cyclic loading conditions 

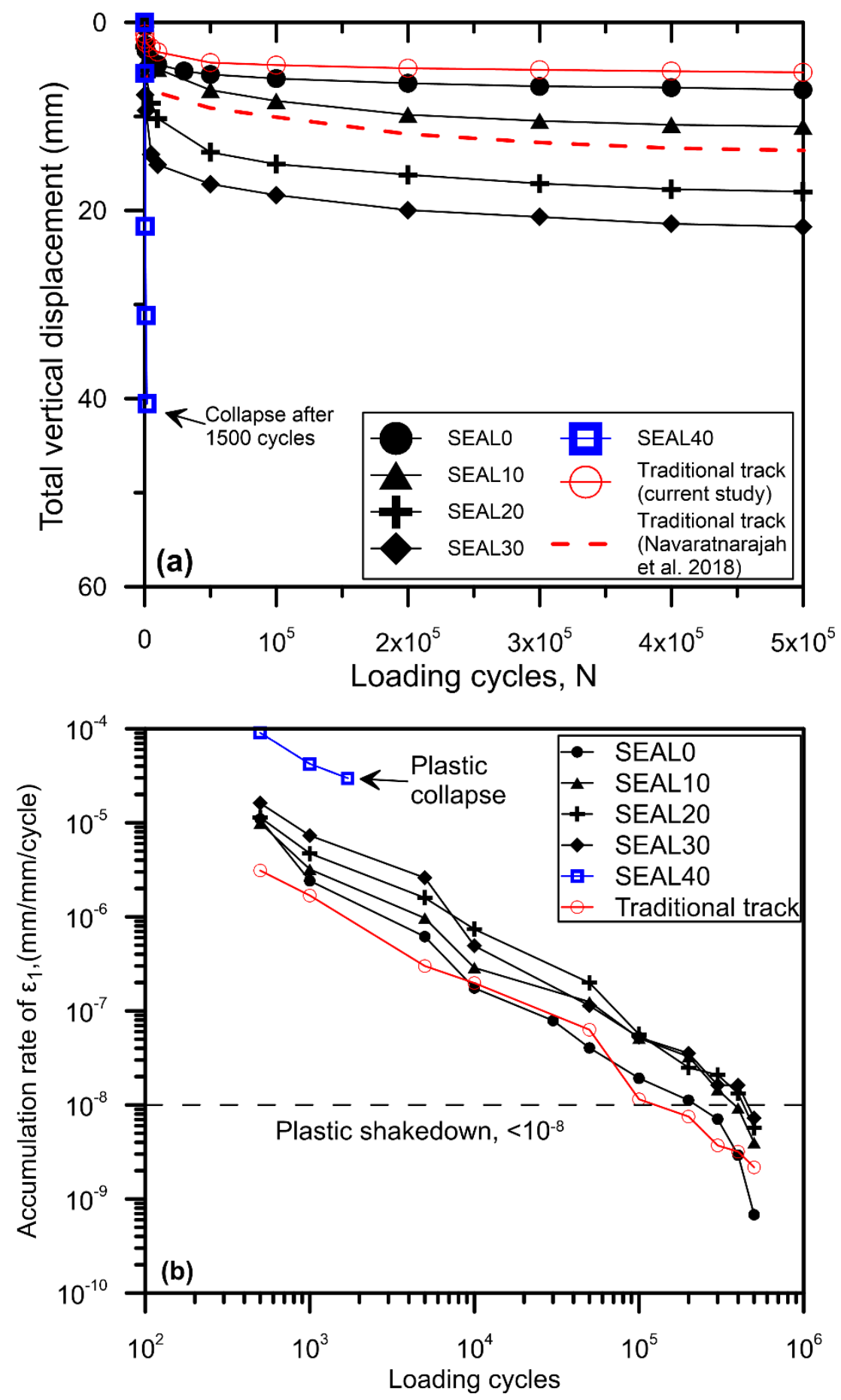

Fig. 3 Deformation responses of the track specimens with different SEAL matrix or traditional materials: (a) vertical displacement, (b) accumulation rate of vertical strain 

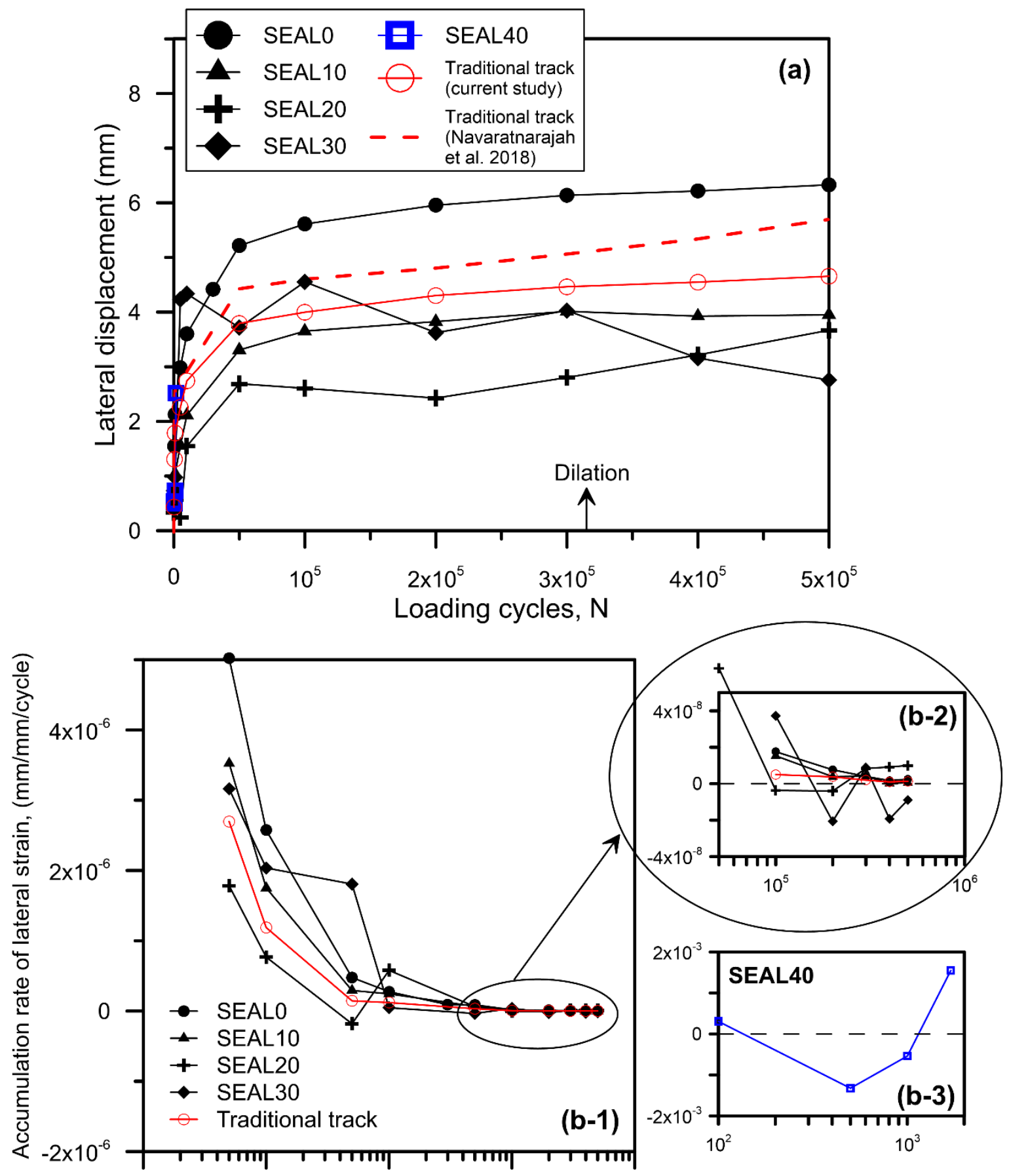

Fig. 4 (a) Lateral displacement of the track specimens with different SEAL matrix and traditional 654 materials; and accumulation rate of lateral strain of track specimens with (b-1, b-2) SEAL0655 SEAL30 and traditional materials, (b-3) SEAL40 

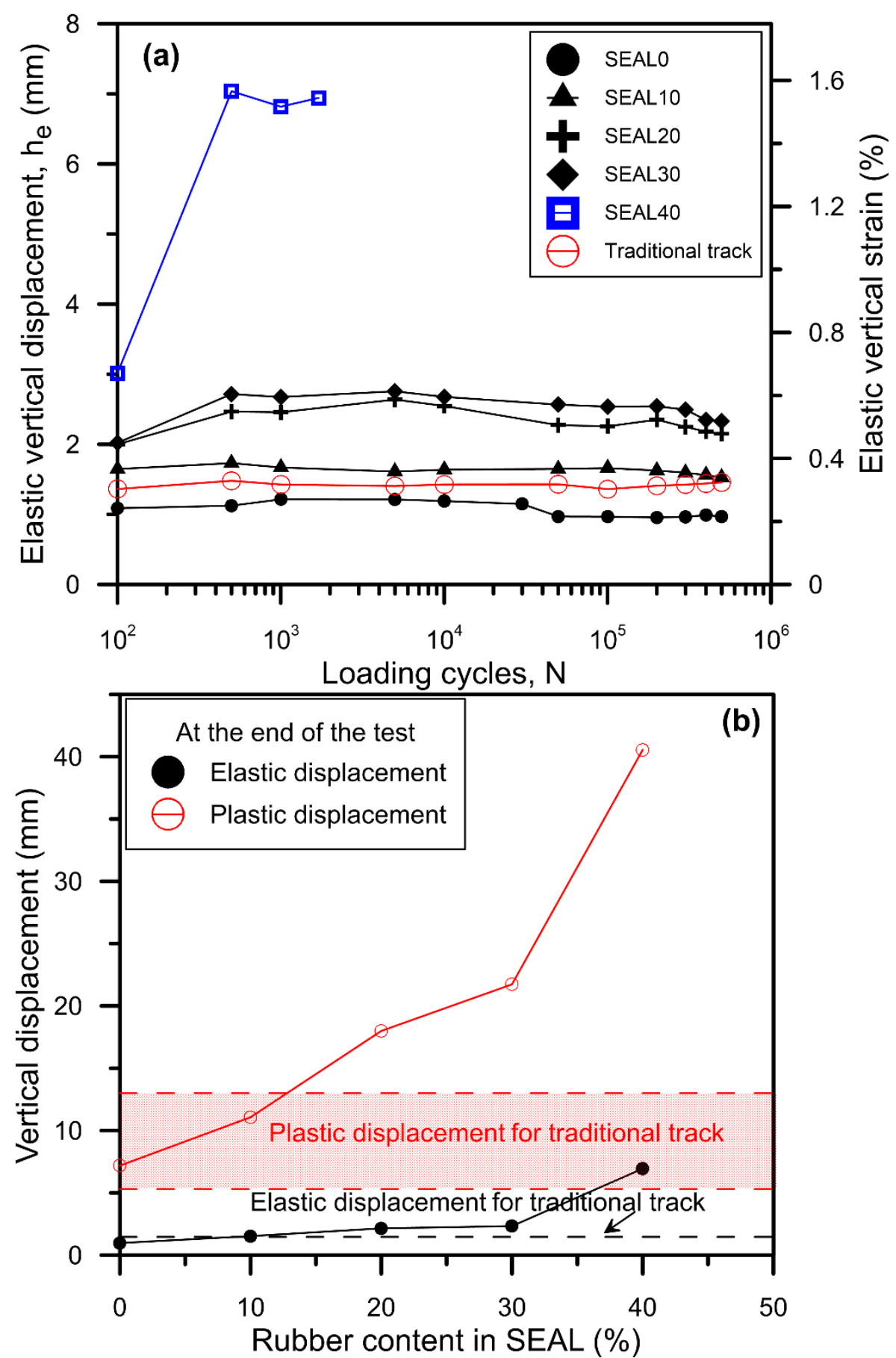

658 Fig. 5 Deformation responses of the track specimens with different SEAL matrix or traditional 659 materials: (a) elastic vertical deformation, and (b) total plastic and elastic deformation 


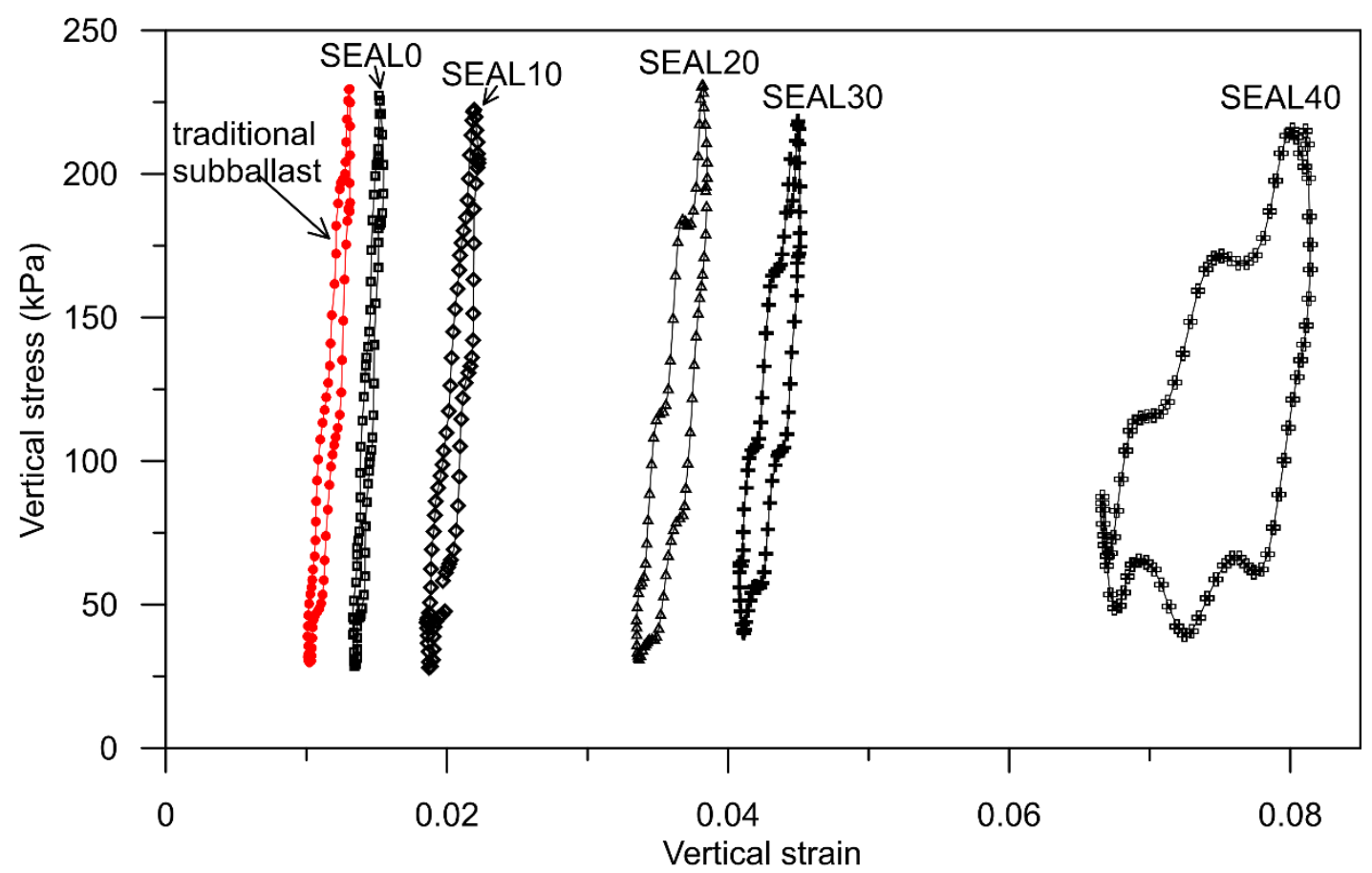

Fig. 6 Hysteretic loops of the track specimen with traditional subballast or SEAL matrix having different rubber contents at the end of each test

665 


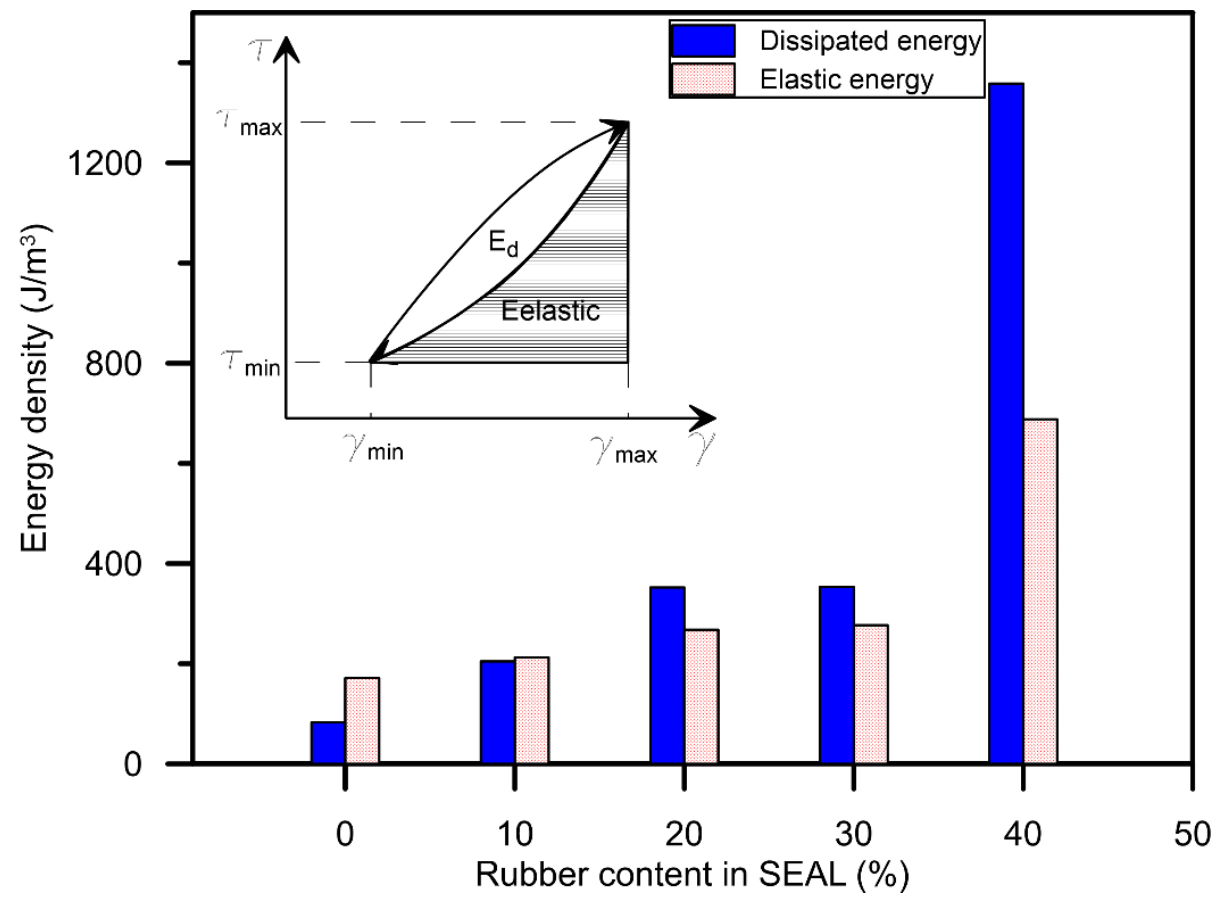

667 Fig. 7 Energy density of the track specimen with SEAL matrix having different rubber contents 668 at the end of each test

669 

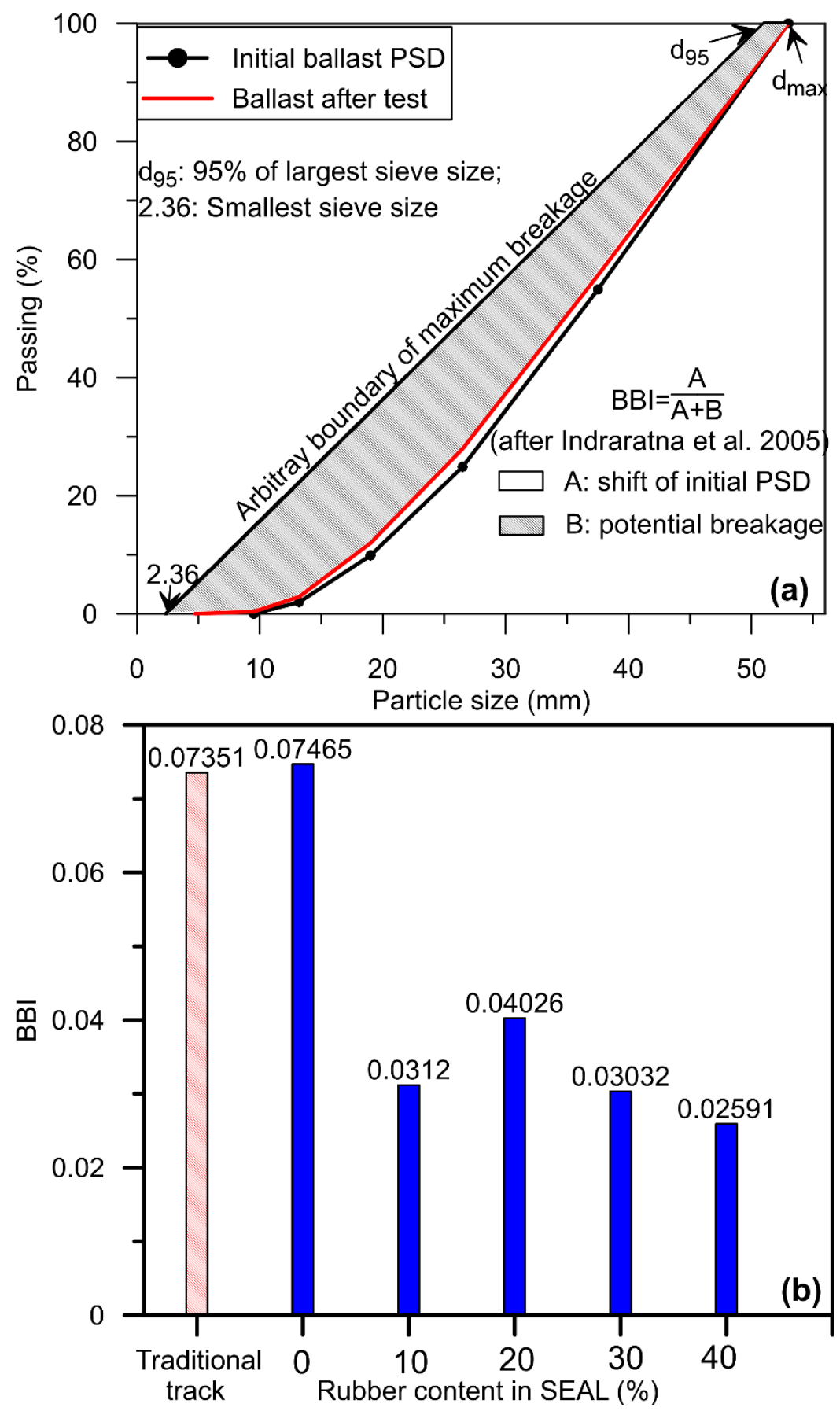

670

671 Fig. 8 (a) Definition of ballast breakage index (BBI); (b) BBI obtained from each test 672 

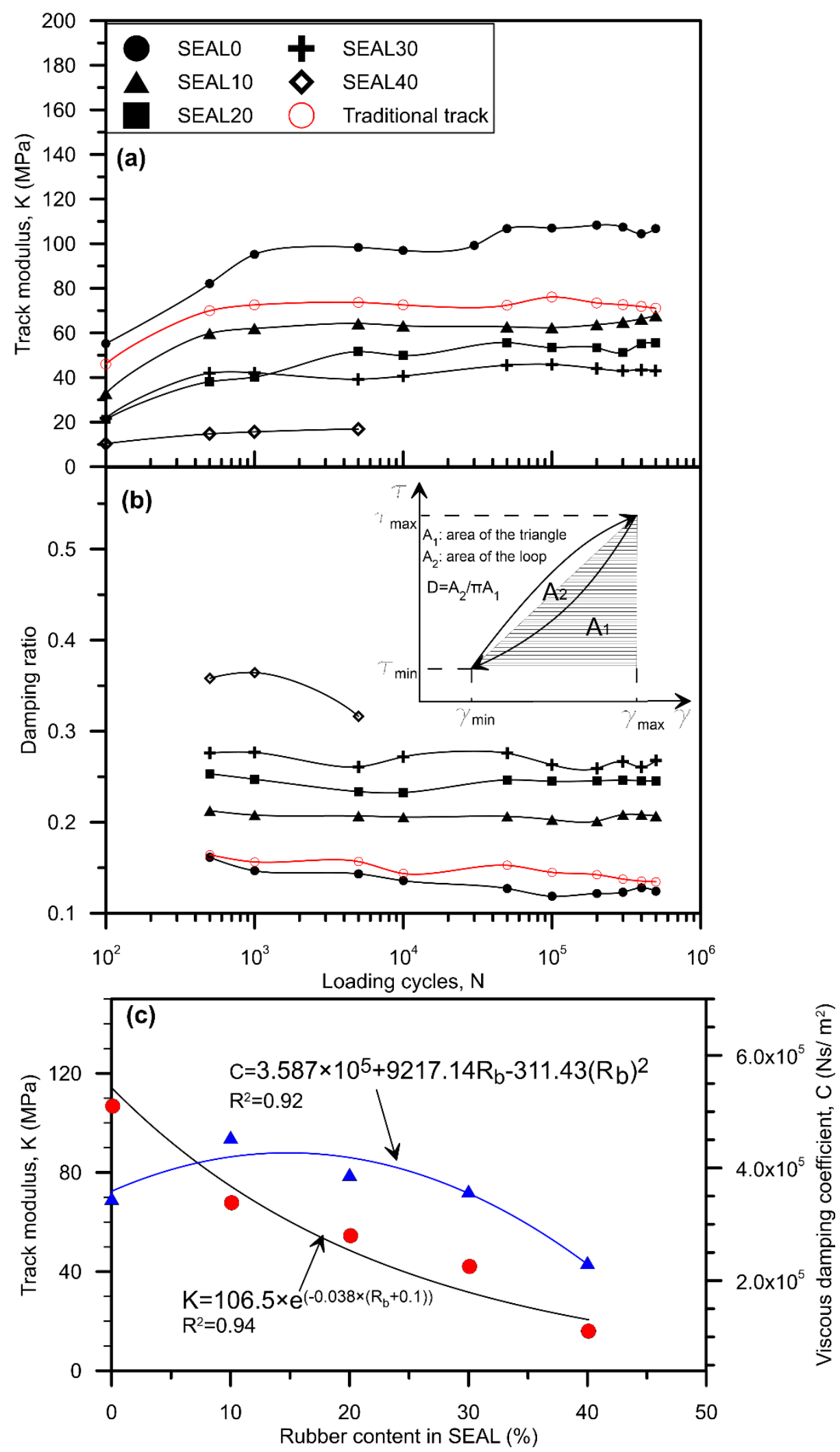

Rubber content in SEAL (\%)

674 Fig. 9 Track modulus and damping ratio of the track specimen with SEAL matrix or traditional 675 subballast materials (a-b) changing with loading cycles, and (c) at the end of each test 

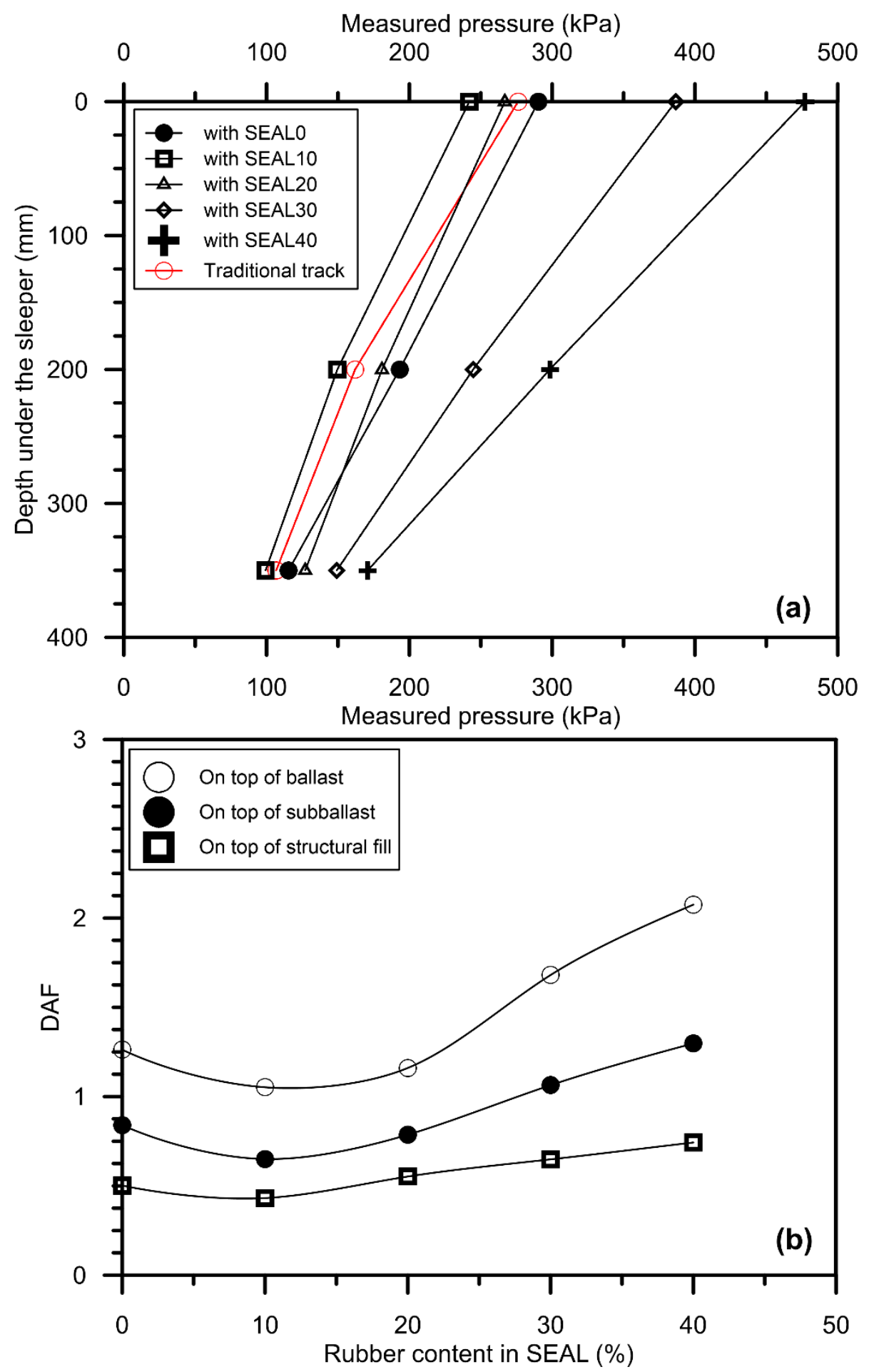

676 Rubber content in SEAL (\%)

677 Fig. 10 (a) Measured pressure on top of each layer (i.e. ballast, subballast and structural fill) for 678 each test; (b) DAF for track specimen changing with RC contents within SEAL matrix 679 
680

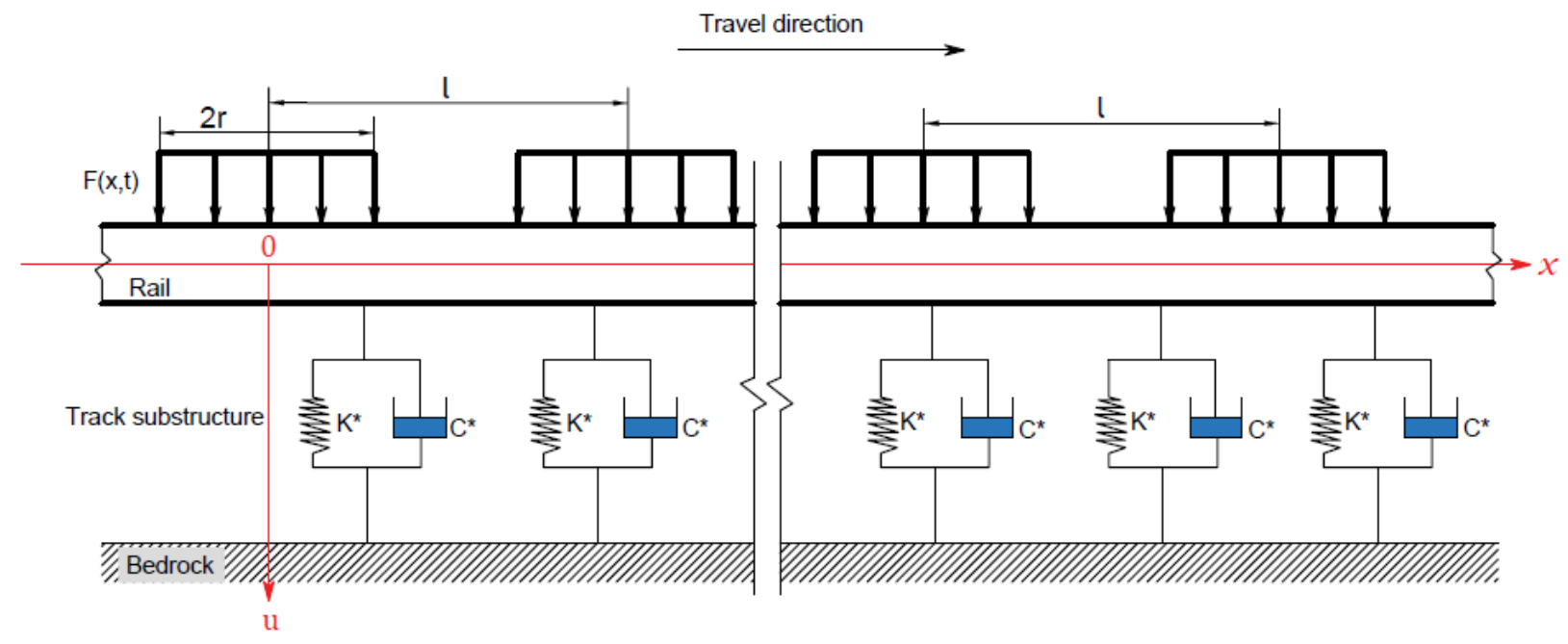

681 Fig. 11 A rail track subjected to a platoon of uniform moving line loads

682 

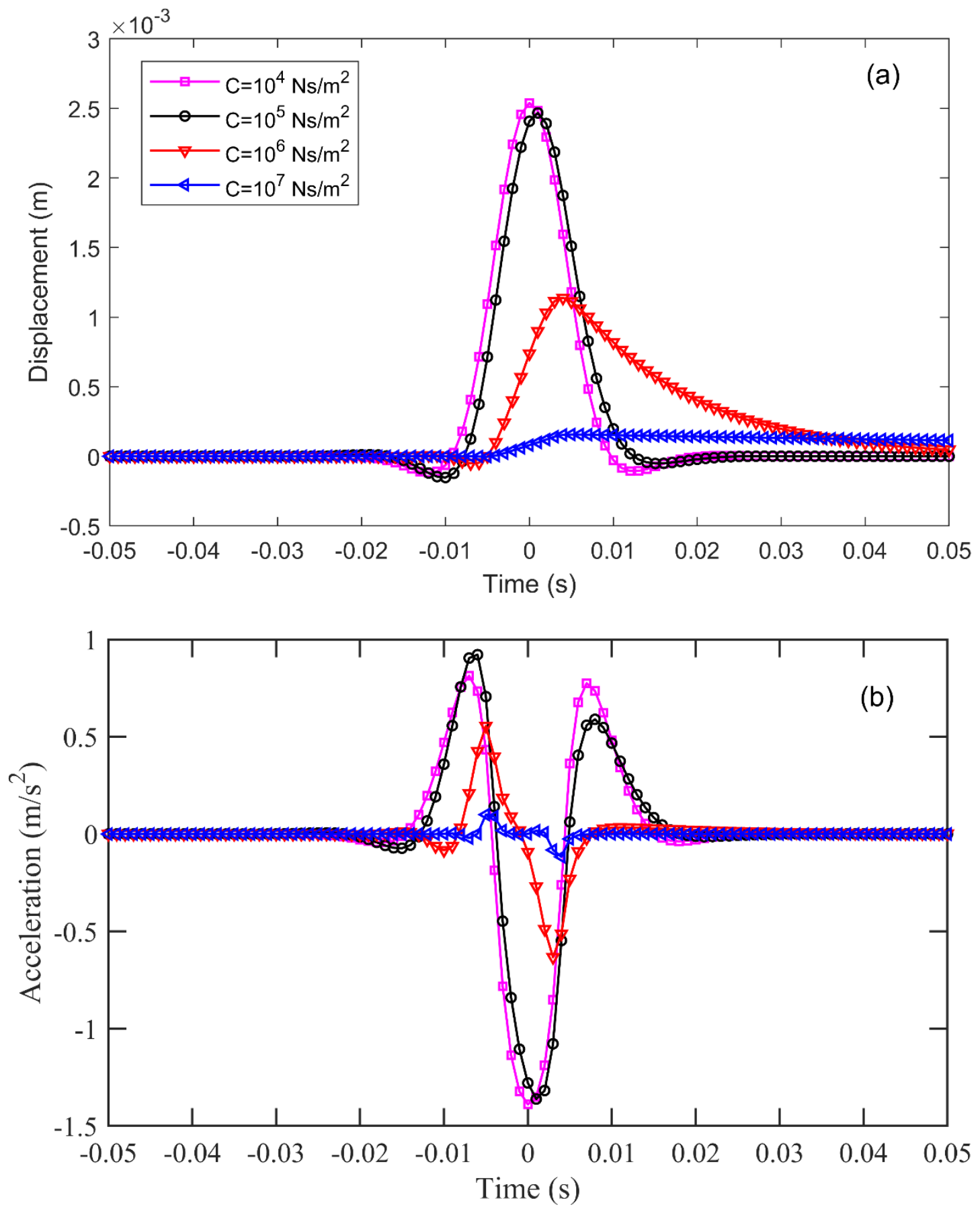

683

684 Fig. 12 Dynamic response of rail with changing viscous damping coefficients: (a) displacement 685 and (b) acceleration 

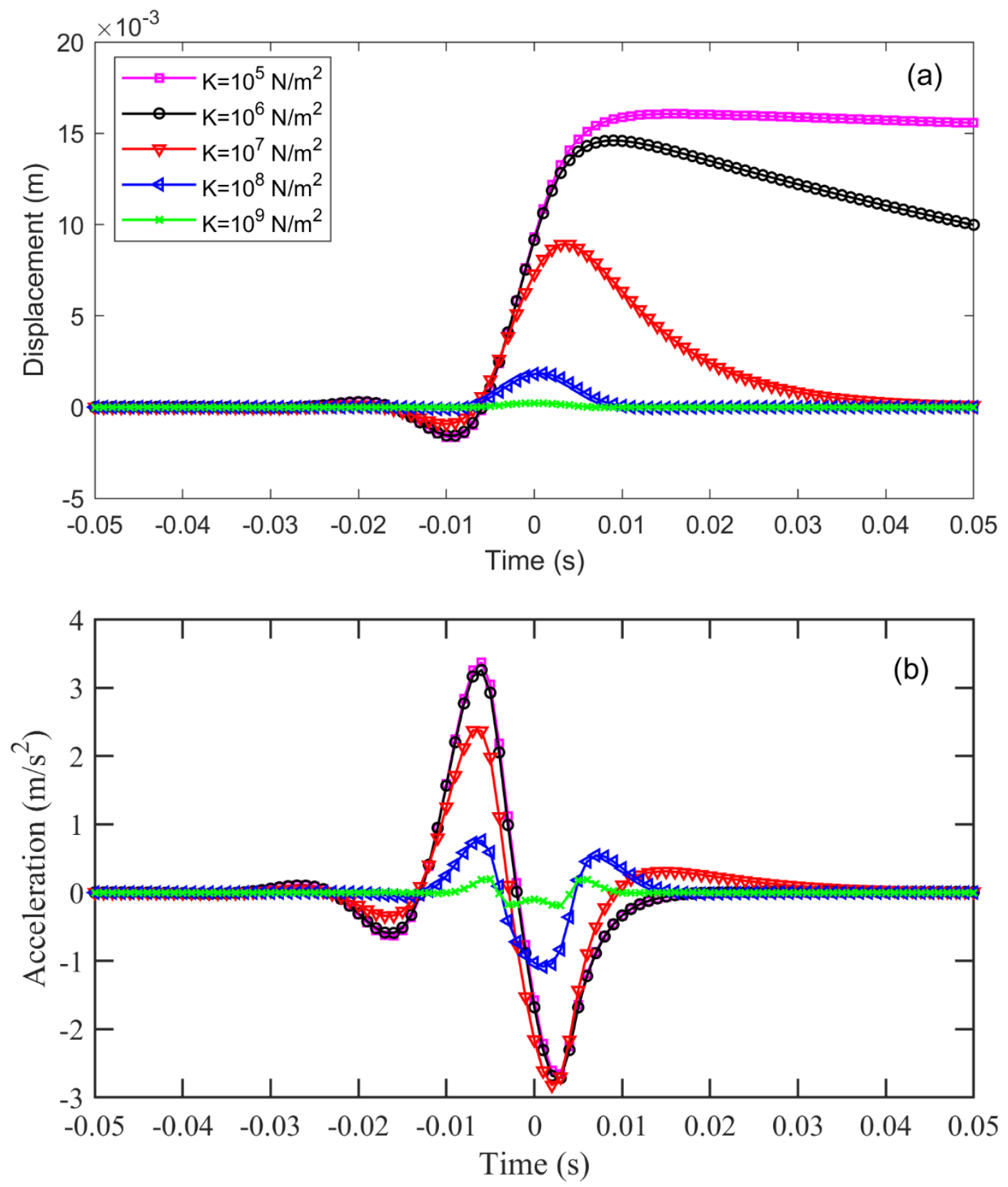

687

688 Fig. 13 Dynamic response of rail with changing shear stiffness: (a) displacement and (b) 689 acceleration 

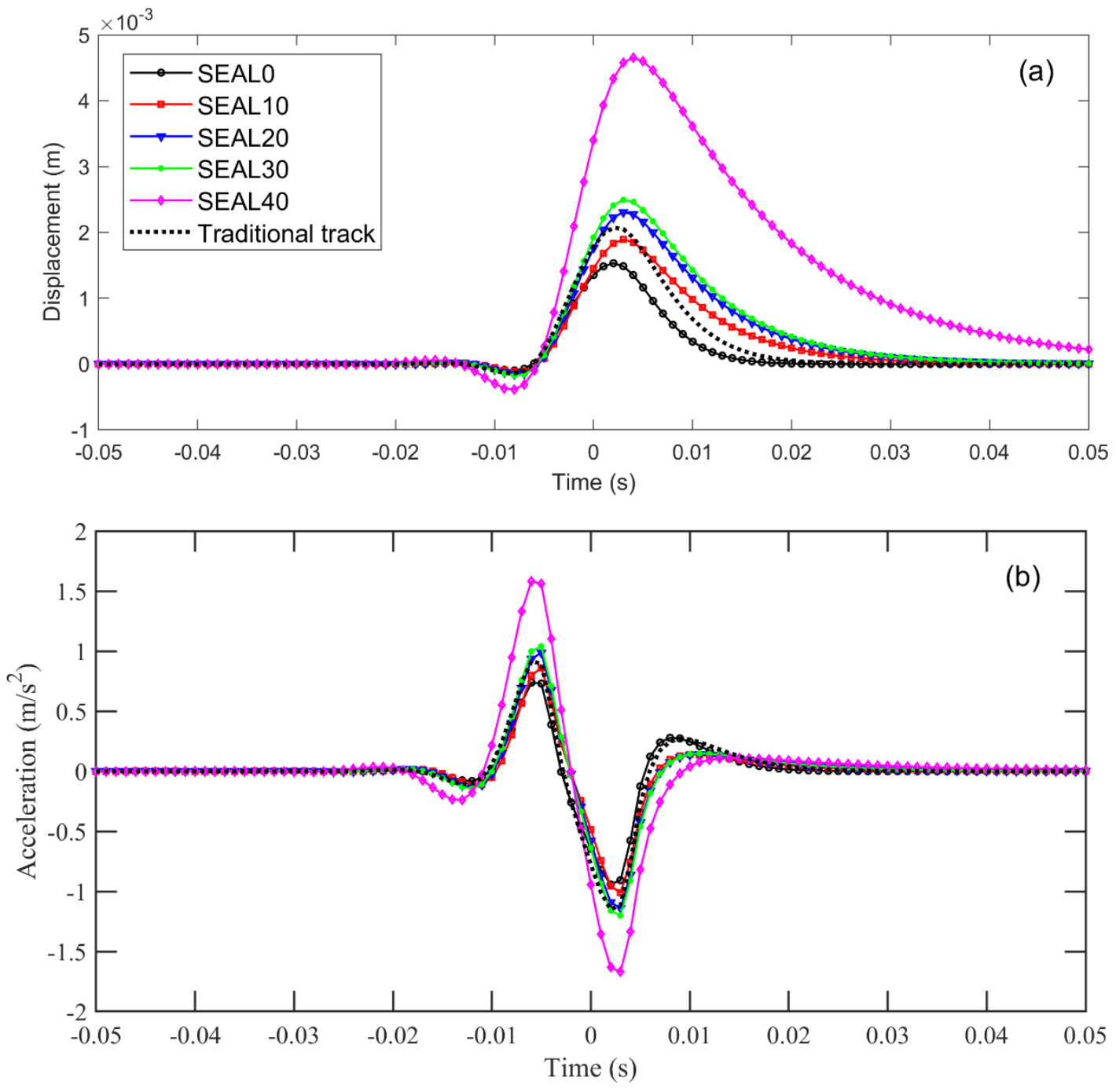

691

692 Fig. 14 Predicted rail dynamic response for traditional track and track incorporated with SEAL 693 (a) displacement and (b) acceleration 

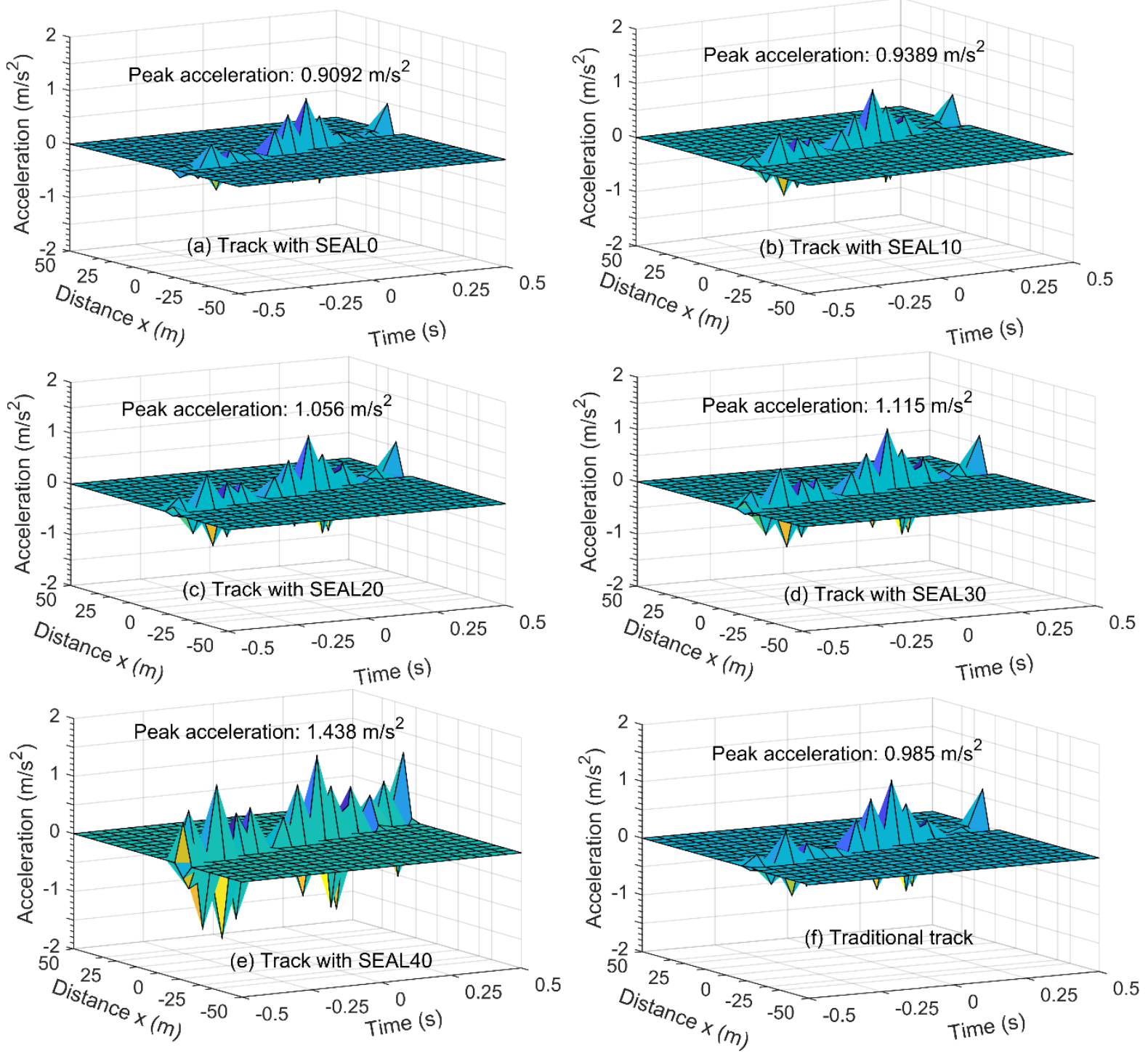

Fig. 15 Predicted 3-D view of the acceleration for the track with (a) SEAL0, (b) SEAL10, (c)

SEAL20, (d) SEAL30 and (e) SEAL40 and (f) traditional materials 